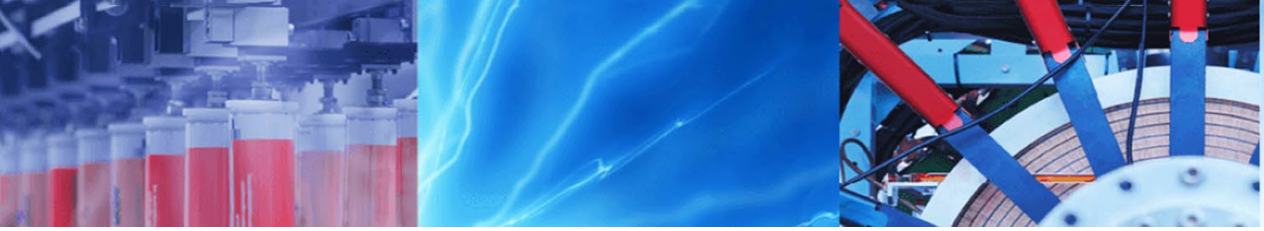

Research Article

\title{
Complex dynamics of delay-induced plankton-fish interaction exhibiting defense
}

\author{
Nilesh Kumar Thakur ${ }^{1}\left[\right.$. Archana Ojha ${ }^{1}$
}

Received: 9 December 2019 / Accepted: 29 April 2020 / Published online: 22 May 2020

(c) Springer Nature Switzerland AG 2020

\begin{abstract}
In this paper, we propose a delayed plankton-fish model with Monod-Haldane-type functional response. Many prey organisms have developed defense mechanisms against predation. Predators also exhibit ways of coping with variable environmental conditions that enter in adaptation in the event of a shortage of prey. We assume prey and middle predators exhibit defense ability towards their predators. Time delay is incorporated in middle predator as an adaptation to cope with variable environmental conditions. Analytically, we study the feasibility and boundedness of the model system by comparison principle followed by local and global stability conditions. We also investigate the stability and direction of bifurcating periodic solution by normal form theory and center manifold arguments. Numerically, we study the effect of defense and time delay on the stability behavior of the system. Our numerical results reveal that the increase in time delay system becomes unstable as well as stable under different parametric restrictions. We observe that due to defense ability in prey and middle predator system shows extinction in top predator.
\end{abstract}

Keywords Plankton · Time delay · Functional response · Defense · Hopf bifurcation

Mathematics Subject Classification 92D25 $34 \mathrm{~K} 20 \cdot 37 \mathrm{M} 05$

\section{Introduction}

The dynamic relationship between predators and their prey has been one of the dominant themes in mathematical ecology. A major concern is to understand how the population of a given species influences the dynamics of other species. Organisms at the lowest trophic levels have to deal with a high risk of predation and to defend themselves against predation which is the main driving force in their evolutionary histories [1]. Planktons are the basis of the aquatic food chain, and its importance for the marine ecosystem is widely recognized [2]. Antigrazer responses of phytoplankton have been widely documented and vary with external abiotic factors [3]. Algal species are highly flexible in their morphology, growth and production of toxin and deterrent compounds and have been interpreted as defense mechanisms against grazing [4]. Production of toxin has great impact on phytoplankton-zooplankton dynamics [5-7]. A study demonstrates that the toxin-producing antipredator defense by phytoplankton may sometimes act as a biocontrol by the stabilizing effect towards the plankton population [8]. Bloom of such algal and fish predation on zooplankton has a strong negative effect on zooplankton and marine ecosystem.

Diel vertical migration (DVM) of zooplankton may be one of the most widely studied defensive strategies [9-11]. But at high predation risk, zooplankton benefits from antipredator defense such as morphological, behavioral, or life history changes to balance the reduction in growth $[12,13]$. The ability to defend from predator attack is an

Nilesh Kumar Thakur, nkthakur.maths@nitrr.ac.in; Archana Ojha, archanaojha1991@gamil.com | Department of Mathematics, National Institute of Technology Raipur, Raipur, CG 492010, India. 
important trait shaping individual fitness, population dynamics and, in consequence, the structure of zooplankton communities [14]. But it has been observed that certain adaptations such as dormancy enable the organism to reduce and manage the environmental stress [15-17]. Adapting dormancy for the evolution of zooplankton is sometimes beneficial to maintain the optimal population size in the environment, i.e., its carrying capacity $[19,20]$. The capability of prey towards the defense provides a new area to analyze the prey-predator model more closely [21, 22]. An experiment shows that at high concentration of filamentous blue-green algae, the reproduction of Daphnia ceases and its population disappears from the environments in few days [23].

One more important parameter that may cause instability and influctuation in stable equilibrium is a time delay relevant for the large number of biological systems; for example, some time is required to digest food by the animals before further activities [24-26]. Time delay affects the stability of positive equilibrium which fluctuates the population causing Hopf bifurcation [28]. It has been observed that time delay has a great influence in any biological process and increasing its value may cause a stable equilibrium to unstable or fluctuation in population $[29,30]$. Rehim and Imran [31] investigated the impact of gestation delay on the phytoplankton-zooplankton system which initially imparts stability and then makes the whole system unstable. Recently, a delayed nutrient cycle model for the plankton system elaborated that delay in decomposition of litter does not influence the stability of the system [32]. Singh and Gakkar [33] observed the rich dynamics including chaos in discrete time delay toxin-producing phytoplankton-zooplankton dynamics. From the last two decades, the researchers have demonstrated very complex models that arise in three or more species food chain models [34-36]. In marine ecosystem, much attention has been paid to top-down and down-top effects on plankton-fish dynamics [37-41]. But the combined study of a time delay with defense (i.e., phytoplankton uses toxicity and zooplankton uses DVM strategy) in plankton-fish interaction is still absent. A number of papers focused on effect of toxic and time delay on the dynamics of plankton system [42-44]. Saha and Bandyopadhyay [45] considered a phytoplankton-zooplankton model system with an additional term which represents extra morality of zooplankton due to toxicity of phytoplankton and studied the phenomenon of stability switching by choosing time delay as a bifurcation parameter. Sharma et al. [46] observed that the digestion time delay has the potential to destabilize the system dynamics in a plankton-fish interaction model. Pal and Chatterjee [47] have discussed the impact of time delay on stability behavior among phytoplankton, zooplankton and fish where the time delay is considered on planktivorous fish due to gestation delay. Liao et al. [48] studied a delayed phytoplankton-zooplankton interaction with Crowley-Martin functional response and revealed that delay can accelerate the procedure of its stability.

Recently, a number of developments have been addressed in the area of population dynamics focused on effect of time delay. Many scholars are excited about solving the system constituting with single or multiple time delays. Chen and Wang [18] investigated the impact of two different time delays on a prey-predator system with Monod-Haldane response function and showed the existence of Hopf bifurcation for possible combinations of two delays. Juneja et al. [27] studied a prey-predator system along with predator harvesting at different harvesting rates through time delay and found the prominent role of time delay in periodic oscillations. Liu and Jiang [37] studied a Gause-type predator-prey model with its biological implications and observed that time delay can switch stability and induced small amplitude oscillations. Time delay in the gestation of the predator is much effective than the feedback time delay of the prey [38]. Kumar et al. [52] explored the complex dynamics by incorporating time delay in the dissemination of information in a SIRS model and discussed its possible insight.

With this motivation, we consider three interacting components consisting of phytoplankton, zooplankton and planktivorous fish in our model system and incorporating discrete-time delay in the dynamics of zooplankton. The main objective of the current study is to closely observe the consequences of time delay on the plankton-fish interaction model where phytoplankton through the production of toxic substances and zooplankton through DVM exhibiting defense towards its predator and the structure of our paper is as follows: In Sect. 2, we have proposed a model system in which time delay is incorporated. Section 3 deals with the analytical methodologies for the proposed model system which includes the positivity and boundedness conditions, equilibria analysis, local stability analysis of positive equilibria and its transversality condition carried out by using its obtained characteristic equation, and at last global stability analysis followed by stability and direction of Hopf bifurcation. In Sect. 4, we have described numerical results to support our analytical findings. In Sect. 5, a discussion is presented for illustrating the biological relevance of our study. Finally, a conclusion is derived in the last section of the paper.

\section{Model formulation}

We consider a model for plankton-fish interaction with Monod-Haldane (MH)-type functional response. The model describes the interaction of prey, specialist predator 
and top specialist predator. At any time $t$, the phytoplankton $x(t)$ is the favorite food of zooplankton $y(t)$, which serves as favorite food for the fish $z(t)$. We assume that the dynamics of the system arise from the coupling of interacting species where fish population $z(t)$ depends only on zooplankton $y(t)$ and zooplankton $y(t)$ on plankton $x(t)$. In the absence of a predator, the prey population grows logistic with carrying capacity $K$ and intrinsic growth rate $r$. Interaction between $(x, y)$ and $(y, z)$ follows MH-type functional response which shows that phytoplankton and zooplankton both are equipped with defense mechanisms. The discrete-time delay has been incorporated in the model system. Under the above consideration, model system satisfies the following:

$$
\begin{aligned}
& \frac{\mathrm{d} x}{\mathrm{~d} t}=r x\left(1-\frac{x}{K}\right)-\frac{c_{1} x y}{b_{1} x^{2}+m_{1}} \\
& \frac{\mathrm{d} y}{\mathrm{~d} t}=-\mathrm{d}_{1} y+\frac{e_{1} c_{1} x(t-\tau) y(t-\tau)}{b_{1} x^{2}(t-\tau)+m_{1}}-\frac{c_{2} y z}{b_{2} y^{2}+m_{2}} \\
& \frac{\mathrm{d} z}{\mathrm{~d} t}=-\mathrm{d}_{2} z+\frac{e_{2} c_{2} y z}{b_{2} y^{2}+m_{2}} .
\end{aligned}
$$

The constant $r$ and $K$ represent the intrinsic growth rate and carrying capacity of prey, respectively. Different parameters used in the model represent different properties for prey and predators; that is, $m_{1}$ and $m_{2}$ are the half-saturation constant of prey and middle predator, $b_{1}$ and $b_{2}$ are the inverse measure of inhibitory effect of middle predator and top predator, $c_{1}$ and $c_{2}$ are the maximum value which can be attained by prey and middle predator for per capita reduction rate, $d_{1}$ and $d_{2}$ are the death rate of middle and top predator, and similarly, $e_{1}$ and $e_{2}$ are the conversion coefficient from individuals of prey to individuals of middle predator and individuals of middle predator to individuals of top predator, respectively.

Here the values of $x, y, z, K, m_{1}$ and $m_{2}$ are measured in number per unit area, values of $r, c_{1}, c_{2}, d_{1}$ and $d_{2}$ are measured in per day, and values of $b_{1}$ and $b_{2}$ are measured in per day ${ }^{-1}$.

The initial conditions of the system (2.1) are given as $x(\theta)=\phi_{1}(\theta), y(\theta)=\phi_{2}(\theta), z(\theta)=\phi_{3}(\theta)$ for all $\theta \in[-\tau, 0]$,

where

$$
\left(\phi_{1}, \phi_{2}, \phi_{3}\right) \in C\left([-\tau, 0], \Re_{+}^{3}\right), \phi_{1}(0), \phi_{2}(0), \phi_{3}(0)>0 .
$$

\section{Analytical methodologies}

\subsection{Positive invariance and boundedness of the solutions}

In this subsection, we demonstrate the positivity and boundedness conditions for the model system (2.1). For this purpose, we define the nonnegative cone by $\mathfrak{R}_{+}^{3}=\left\{(x, y, z) \in \mathfrak{R}_{+}^{3} \mid x \geq 0 ; y \geq 0 ; z \geq 0\right\}$ and positive cone by int $\left(\mathfrak{R}_{+}^{3}\right)=\left\{(x, y, z) \in \mathfrak{R}_{+}^{3} \mid x>0 ; y>0 ; z>0\right\}$.

Theorem 1 The positive cone for the system (2.1) with initial conditions is invariant.

Proof To show the positivity condition, we have from the system (2.1),

$$
\begin{aligned}
x(t)= & x(0) \exp \left\{\int_{0}^{t}\left(r\left(1-\frac{x(s)}{K}\right)-\frac{c_{1} y(s)}{b_{1} x^{2}(s)+m_{1}}\right) \mathrm{d} s\right\}, \\
y(t)= & y(0) \exp \left\{\int _ { 0 } ^ { t } \left(-d_{1}+\frac{e_{1} c_{1} x(s-\tau) y(s-\tau)}{y(s)\left(b_{1} x^{2}(s-\tau)+m_{1}\right)}\right.\right. \\
& \left.\left.-\frac{c_{2} z(s)}{b_{2} y^{2}(s)+m_{2}}\right) \mathrm{~d} s\right\}, \\
z(t)= & z(0) \exp \left\{\int_{0}^{t}\left(-d_{2}+\frac{e_{2} c_{2} y(s)}{b_{2} y^{2}(s)+m_{2}}\right) \mathrm{d} s\right\},
\end{aligned}
$$

which shows that, $x(t)>0, y(t)>0, z(t)>0$ if $x(0)>0$, $y(0)>0, z(0)>0$. Therefore, the positive cone $\mathfrak{R}_{+}^{3}$ is an invariant region.

Theorem 2 Let $\Omega(t)=(x(t), y(t), z(t))$ be any positive solution of the system (2.1), then there exists $a T>0$ such that $0 \leq x(t) \leq M, 0 \leq y(t) \leq M^{\prime}$ and $0 \leq z(t) \leq M^{\prime \prime}$, for $t>T$, where

$$
\begin{aligned}
M & =K, \\
M^{\prime} & =\frac{e_{1} r K}{4 d_{1}} \\
M^{\prime \prime} & =e_{2}\left(\frac{e_{1} r K}{4 b}-M^{\prime}\right) .
\end{aligned}
$$

Proof We already proved $(x(t), y(t), z(t))$ remains nonnegative in Theorem 1. So we only need to show that $x(t) \leq M$, $y(t) \leq M^{\prime}$ and $z(t) \leq M^{\prime \prime}$.

From the first equation of the system (2.1), we have

$$
\frac{\mathrm{d} x}{\mathrm{~d} t} \leq r x\left(1-\frac{x}{K}\right), x(0)>0,
$$

thus, by simple calculation, we get

$\lim _{t \rightarrow \infty} \sup x(t) \leq K=M$. 
Now we define a function

$\chi(t)=x(t-\tau)+\frac{y(t)}{e_{1}}$.

Differentiating with respect to $t$, we get

$$
\begin{aligned}
\frac{\mathrm{d} \chi}{\mathrm{d} t}= & \frac{\mathrm{d} x(t-\tau)}{\mathrm{d} t}+\frac{1}{e_{1}} \frac{\mathrm{d} y(t)}{\mathrm{d} t}, \\
= & r x(t-\tau)\left(1-\frac{x(t-\tau)}{K}\right)-\frac{c_{1} x(t-\tau) y(t-\tau)}{b_{1} x^{2}(t-\tau)+m_{1}} \\
& -\frac{d_{1}}{e_{1}} y(t)+\frac{c_{1} x(t-\tau) y(t-\tau)}{b_{1} x^{2}(t-\tau)+m_{1}} \\
& -\frac{c_{2} y(t) z(t)}{e_{1}\left(b_{2} y^{2}(t)+m_{2}\right)}, \\
\frac{\mathrm{d} \chi}{\mathrm{d} t} \leq & r x(t-\tau)\left(1-\frac{x(t-\tau)}{K}\right)-\frac{d_{1}}{e_{1}} y(t)-d_{1} x(t-\tau) \\
& +d_{1} x(t-\tau), \\
\frac{\mathrm{d} \chi}{\mathrm{d} t}+ & d_{1} \chi(t) \leq \frac{r K}{4}+d_{1} K, \text { since, } \max x\left(1-\frac{x}{K}\right)=\frac{K}{4} .
\end{aligned}
$$

By comparison lemma for $t \geq \tilde{T} \geq 0$, we obtain

$\chi(t) \leq \frac{r K}{4 d_{1}}+K-\left(\frac{r K}{4 d_{1}}+K-\chi(\tilde{T})\right) \mathrm{e}^{-d_{1}(t-\tilde{T})}$,

If $\tilde{T}=0$, then

$\chi(t) \leq \frac{r K}{4 d_{1}}+K-\left(\frac{r K}{4 d_{1}}+K-\chi(0)\right) \mathrm{e}^{-d_{1} t}$.

For the large value of $t$, we have

$$
\begin{aligned}
x(t-\tau)+\frac{y(t)}{e_{1}} & \leq \frac{r K}{4 d_{1}}+K, \\
\frac{y(t)}{e_{1}} & \leq \frac{r K}{4 d_{1}} .
\end{aligned}
$$

Therefore,

$y(t) \leq \frac{e_{1} r K}{4 d_{1}}=M^{\prime}$.

Again, we define a function

$\rho(t)=e_{1} x(t-\tau)+y(t)+\frac{z(t)}{e_{2}}$.

Differentiating with respect to $t$, we get

$$
\begin{aligned}
\frac{\mathrm{d} \rho}{\mathrm{d} t}= & e_{1} \frac{\mathrm{d} x(t-\tau)}{\mathrm{d} t}+\frac{d y(t)}{\mathrm{d} t}+\frac{1}{e_{2}} \frac{\mathrm{d} z(t)}{\mathrm{d} t}, \\
= & e_{1} r x(t-\tau)\left(1-\frac{x(t-\tau)}{K}\right) \\
& -\frac{e_{1} c_{1} x(t-\tau) y(t-\tau)}{b_{1} x^{2}(t-\tau)+m_{1}}-d_{1} y(t)+\frac{e_{1} c_{1} x(t-\tau) y(t-\tau)}{b_{1} x^{2}(t-\tau)+m_{1}} \\
& -\frac{c_{2} y(t) z(t)}{b_{2} y^{2}(t)+m_{2}}-\frac{d_{2}}{e_{2}} z(t)+\frac{c_{2} y(t) z(t)}{b_{2} y^{2}(t)+m_{2}} \\
\frac{\mathrm{d} \rho}{\mathrm{d} t}= & e_{1} r x(t-\tau)\left(1-\frac{x(t-\tau)}{K}\right)-d_{1} y(t)-\frac{d_{2}}{e_{2}} z(t), \\
\frac{\mathrm{d} \rho}{\mathrm{d} t} \leq & \frac{e_{1} r K}{4}-d_{1} y(t)-\frac{d_{2}}{e_{2}} z(t), \text { since, } \max x\left(1-\frac{x}{K}\right)=\frac{K}{4} .
\end{aligned}
$$

Taking $b=\min \left\{d_{1}, d_{2}\right\}$, which implies

$\frac{\mathrm{d} \rho}{\mathrm{d} t} \leq \frac{e_{1} r K}{4}-b\left(y(t)+\frac{z(t)}{e_{2}}\right)-b e_{1} x(t-\tau)+b e_{1} x(t-\tau)$,

$\frac{\mathrm{d} \rho}{\mathrm{d} t}+b \rho \leq \frac{e_{1} r K}{4}+b e_{1} K$.

By comparison lemma for $t \geq \tilde{T} \geq 0$, we obtain

$\rho \leq \frac{e_{1} r K}{4 b}+e_{1} K-\left(\frac{e_{1} r K}{4 b}+e_{1} K-\rho(\tilde{T})\right) \mathrm{e}^{-b(t-\tilde{T})}$,

If $\tilde{T}=0$, then

$\rho \leq \frac{e_{1} r K}{4 b}+e_{1} K-\left(\frac{e_{1} r K}{4 b}+e_{1} K-\rho(0)\right) \mathrm{e}^{-b t}$,

For the large value of $t$, we have

$$
\begin{aligned}
e_{1} x(t-\tau)+y(t)+\frac{z(t)}{e_{2}} & \leq \frac{e_{1} r K}{4 b}+e_{1} K, \\
y(t)+\frac{z(t)}{e_{2}} & \leq \frac{e_{1} r K}{4 b} .
\end{aligned}
$$

Therefore,

$z(t) \leq e_{2}\left(\frac{e_{1} r K}{4 b}-M^{\prime}\right)=M^{\prime \prime}$ such that $e_{1} r K>4 b M^{\prime}$.

Thus all species are uniformly bounded for initial value in $\mathfrak{R}_{+}^{3}$. This completes the proof of Theorem 2 .

\subsection{Equilibrium analysis}

In this subsection, the existence of possible equilibria and stability condition corresponding to the different equilibrium points is investigated. It is clear that model system (2.1) possesses four nonnegative equilibrium points, namely $E_{0}(0,0,0), E_{x}(K, 0,0), E_{x y}\left(x_{3}, y_{3}, 0\right)$ and $E^{*}\left(x^{*}, y^{*}, z^{*}\right)$. 
(i) Existence of $E_{0}(0,0,0)$ as trivial equilibrium point. The eigenvalues of the variational matrix around $E_{0}$ are $r_{1}-d_{1}$ and $-d_{2}$. The variational matrix has a positive root and two negative roots. Hence, $E_{0}$ is a is saddle point with two-dimensional stable manifold in yz-plane and one-dimensional unstable manifold in $x$-direction.

(ii) Existence of $E_{x}(K, 0,0)$ as predator free equilibrium point. The eigenvalues of the variational matrix around $E_{x}$ are $-r_{1}-d_{1}+\frac{e_{1} c_{1} K}{b_{1} K^{2}+m_{1}}$ and $-d_{2}$. The variational matrix has two negative roots. Hence, $E_{x}$ is also a saddle point with two-dimensional stable manifold in $x z$-plane and one-dimensional unstable manifold in $y$-direction if $e_{1} c_{1} K>d_{1}\left(b_{1} K^{2}+m_{1}\right)$. $E_{x}$ is locally asymptotically stable, if $e_{1} c_{1} K<d_{1}\left(b_{1} K^{2}+m_{1}\right)$.

(iii) Existence of $E_{x y}\left(x_{3}, y_{3}, 0\right)$ as top-predator free equilibrium point, where $x_{3}$ and $y_{3}$ are obtained by the following relations;

$x_{3}=\frac{\frac{e_{1} c_{1}}{b_{1} d_{1}} \pm \sqrt{\left(\frac{e_{1} c_{1}}{b_{1} d_{1}}\right)^{2}-\frac{4 m_{1}}{b_{1}}}}{2}$

$y_{3}=\frac{r}{c_{1}}\left(1-\frac{x_{3}}{K}\right)\left(b_{1} x_{3}^{2}+m_{1}\right)$.

If $\left(\frac{e_{1} c_{1}}{b_{1} d_{1}}\right)^{2} \geq \frac{4 m_{1}}{b_{1}}$ then $E_{x y}$ is positive and real, provided $K>x_{3}$. The variational matrix around $E_{x y}$ satisfies the following:

$$
\begin{aligned}
\lambda_{1}+\lambda_{2} & =-\frac{r x_{3}}{K}+\frac{2 b_{1} c_{1} x_{3}^{2} y_{3}}{\left(b_{1} x_{3}^{2}+m_{1}\right)^{2}}, \\
\lambda_{1} \lambda_{2} & =\frac{\left(m_{1}-b_{1} x_{3}^{2}\right) e_{1} c_{1}^{2} x_{3} y_{3}}{\left(b_{1} x_{3}^{2}+m_{1}\right)^{2}}, \\
\lambda_{3} & =-d_{2}+\frac{e_{2} c_{2} y_{3}}{b_{2} y_{3}^{2}+m_{2}} .
\end{aligned}
$$

Two eigenvalues $\lambda_{1}$ and $\lambda_{2}$ have negative real parts if $\lambda_{1}+\lambda_{2}<0$ and $\lambda_{1} \lambda_{2}>0$. Hence, the equilibrium point $E_{x y}$ is locally asymptotically stable, if $\frac{r x_{3}}{K}>\frac{2 b_{1} c_{1} x_{3}^{2} y_{3}}{\left(b_{1} x_{3}^{2}+m_{1}\right)^{2}}, m_{1}-b_{1} x_{3}^{2}>0$ and $\frac{e_{2} c_{2} y_{3}}{b_{2} y_{3}^{2}+m_{2}}>d_{2}$ holds.

(iv) Existence of coexistence equilibrium point $E^{*}\left(x^{*}, y^{*}, z^{*}\right)$, where $x^{*}$ is the positive root of the following equation

$$
\begin{aligned}
& x^{* 3}-K x^{* 2}+\frac{m_{1}}{b_{1}} x^{*}+\frac{K}{b_{1}}\left(\frac{c_{1} y^{*}}{r}-m_{1}\right)=0 . \\
& \text { Let } f\left(x^{*}\right)=x^{* 3}-K x^{* 2}+\frac{m_{1}}{b_{1}} x^{*}+\frac{K}{b_{1}}\left(\frac{c_{1} y^{*}}{r}-m_{1}\right), \\
& \text { then } f(0)=\frac{K}{b_{1}}\left(\frac{c_{1} y^{*}}{r}-m_{1}\right), f(0)<0 \text { if } y^{*}<\frac{m_{1} r}{c_{1}} \text { and }
\end{aligned}
$$

$f(K)=\frac{c_{1} K y^{*}}{r b_{1}}>0$. Since $f(0) f(K)<0$, by intermediate value theorem, Eq. (3.1) has a positive root lies in $(0, K)$ when

$$
y^{*}<\frac{m_{1} r}{c_{1}} \text {. }
$$

$y^{*}$ is a positive root of the quadratic equation

$$
g\left(y^{*}\right)=y^{*}-\frac{e_{2} c_{2}}{b_{2} d_{2}} y^{*}+\frac{m_{2}}{b_{2}}=0 .
$$

Roots of $g\left(y^{*}\right)=0$ take the form $y^{*}=\frac{\frac{e_{2} c_{2}}{b_{2} d_{2}} \pm \sqrt{\left(\frac{e_{2} c_{2}}{b_{2} d_{2}}\right)^{2}-\frac{4 m_{2}}{b_{2}}}}{2}$, out of which at least one will be positive provided one of the following conditions holds:
(a) $\frac{e_{2} c_{2}}{b_{2} d_{2}}>0$ and $\frac{m_{2}}{b_{2}}<0$,
(b) $\frac{e_{2} c_{2}}{b_{2} d_{2}}>0, \frac{m_{2}}{b_{2}}>0$ and $\left(\frac{e_{2} c_{2}}{b_{2} d_{2}}\right)^{2}-\frac{4 m_{2}}{b_{2}}>0$,
(c) $\frac{e_{2} c_{2}}{b_{2} d_{2}}<0$ and $\frac{m_{2}}{b_{2}}<0$.

$z^{*}$ is given by the equation

$$
z^{*}=\frac{1}{c_{2}}\left(-d_{1}+\frac{e_{1} c_{1} x^{*}}{b_{1} x^{* 2}+m_{1}}\right)\left(b_{2} y^{* 2}+m_{2}\right)
$$

where $z^{*}>0$ if

$$
d_{1}<\frac{e_{1} c_{1} x^{*}}{b_{1} x^{* 2}+m_{1}}
$$

Hence, the coexistence equilibrium $E^{*}\left(x^{*}, y^{*}, z^{*}\right)$ exists if the conditions (3.2), (3.4) and (3.6) hold.

\subsection{Local stability analysis about $E^{*}\left(x^{*}, y^{*}, z^{*}\right)$}

Now we focus on the study of local stability around the positive equilibria $E^{*}\left(x^{*}, y^{*}, z^{*}\right)$ and occurrence of Hopf bifurcation of the system (2.1). For this, let $x(t)=x^{*}+X(t)$, $y(t)=y^{*}+Y(t)$ and $z(t)=z^{*}+Z(t)$. This helps to perturb the system (2.1) and we get a new system of equations as follows:

$\frac{d}{d t}\left(\begin{array}{l}X(t) \\ Y(t) \\ Z(t)\end{array}\right)=J\left(\begin{array}{l}X(t) \\ Y(t) \\ Z(t)\end{array}\right)+J^{\prime}\left(\begin{array}{l}X(t-\tau) \\ Y(t-\tau) \\ Z(t-\tau)\end{array}\right)$,

where 
$J=\left(\begin{array}{lll}a & b & 0 \\ 0 & c & d \\ 0 & e & 0\end{array}\right)$ and $J^{\prime}=\left(\begin{array}{lll}0 & 0 & 0 \\ f & g & 0 \\ 0 & 0 & 0\end{array}\right)$

in which

$a=-\frac{r x^{*}}{K}+\frac{2 b_{1} c_{1} x^{* 2} y^{*}}{\left(b_{1} x^{* 2}+m_{1}\right)^{2}}$

$b=-\frac{c_{1} x^{*}}{b_{1} x^{* 2}+m_{1}}$

$c=-d_{1}-\frac{\left(m_{2}-b_{2} y^{* 2}\right) c_{2} z^{*}}{\left(b_{2} y^{* 2}+m_{2}\right)^{2}}$

$d=-\frac{c_{2} y^{*}}{b_{2} y^{* 2}+m_{2}}$

$e=\frac{\left(m_{2}-b_{2} y^{* 2}\right) e_{2} c_{2} z^{*}}{\left(b_{2} y^{* 2}+m_{2}\right)^{2}}$

$f=\frac{\left(m_{1}-b_{1} x^{* 2}\right) e_{1} c_{1} y^{*}}{\left(b_{1} x^{* 2}+m_{1}\right)^{2}}$

$g=\frac{e_{1} c_{1} x^{*}}{b_{1} x^{* 2}+m_{1}}$.

The characteristic equation of the delayed system (2.1) takes the form

$\lambda^{3}+A \lambda^{2}+B \lambda+C+\mathrm{e}^{-\lambda \tau}\left(D \lambda^{2}+E \lambda\right)=0$,

where

$A=-(a+c)$,

$B=a c-d e$,

$C=$ ade,

$D=-g$,

$E=a g-b f$.

When $\tau=0$, we get the characteristic equation of delay free system, which is given by

$\lambda^{3}+(A+D) \lambda^{2}+(B+E) \lambda+C=0$.

Therefore, the stability conditions for $E^{*}\left(x^{*}, y^{*}, z^{*}\right)$ are defined by the following hypothesis;

\section{Hypothesis $\left(H_{1}\right)$}

$(A+D)>0, C>0$,

\section{Hypothesis $\left(\mathrm{H}_{2}\right)$}

$(A+D)(B+E)-C>0$.

Then, by Routh-Hurwitz criterion, we state that:
Lemma 1 If Hypotheses $\left(H_{1}\right)$ and $\left(H_{2}\right)$ hold, then all the roots of Eq. (3.9) have negative real parts which show that the coexistence equilibrium $E^{*}\left(x^{*}, y^{*}, z^{*}\right)$ is locally asymptotically stable for $\tau=0$.

For occurrence of Hopf bifurcation, it can be assumed that Eq. (3.8) has a pair of purely imaginary roots. Let this root be $\lambda=i \omega_{0}, \tau=\tau_{0}$ and putting this into Eq. (3.8), for simplicity, referring $\omega_{0}$ and $\tau_{0}$ by $\omega, \tau$, respectively, we obtained

$$
\begin{aligned}
& -i \omega^{3}-A \omega^{2}+i B \omega+C-D \omega^{2} \cos \omega \tau+i E \omega \cos \omega \tau \\
& +i D \omega^{2} \sin \omega \tau+E \omega \sin \omega \tau=0 .
\end{aligned}
$$

Separating the real and imaginary parts, we have

$-A \omega^{2}+C=D \omega^{2} \cos \omega \tau-E \omega \sin \omega \tau$,

$-\omega^{3}+B \omega=-E \omega \cos \omega \tau-D \omega^{2} \sin \omega \tau$.

Now, squaring and adding leads the following six-degree equation;

$\omega^{6}+\left(A^{2}-2 B-D^{2}\right) \omega^{4}+\left(B^{2}-2 A C-E^{2}\right) \omega^{2}+C^{2}=0$.

By simple algebraic calculation and taking $u=\omega^{2}$, Eq. (3.13) becomes

$u^{3}+Q_{1} u^{2}+Q_{2} u+Q_{3}=0$,

where

$Q_{1}=\left(A^{2}-2 B-D^{2}\right)$,

$Q_{2}=\left(B^{2}-2 A C-E^{2}\right)$,

$Q_{3}=C^{2}$.

For the roots distribution, let

$H(u)=u^{3}+Q_{1} u^{2}+Q_{2} u+Q_{3}$

then

$H^{\prime}(u)=3 u^{2}+2 Q_{1} u+Q_{2}$.

Hence, $H^{\prime}(u)=0$ has two roots, which are given by

$$
\begin{aligned}
& u_{1}^{*}=\frac{-Q_{1}+\sqrt{\left(Q_{1}^{2}-3 Q_{2}\right)}}{3}, \\
& u_{2}^{*}=\frac{-Q_{1}-\sqrt{\left(Q_{1}^{2}-3 Q_{2}\right)}}{3} .
\end{aligned}
$$


Now, we make the hypothesis which is given below;

\section{Hypothesis $\left(H_{3}\right)$}

$u_{1}^{*}>0, H\left(u_{1}^{*}\right)<0, Q_{1}^{2}-3 Q_{2} \geqslant 0$.

Since $Q_{3}>0$, Eq. (3.14) has no real positive roots if $Q_{1}-3 Q_{2}<0$ (see lemma 2.1 in [49]) and two real positive roots if Hypothesis $\left(H_{3}\right)$ holds and these roots be $\omega_{j}(j=1,2)$. Let $\omega_{1}<\omega_{2}$ then $H^{\prime}\left(\omega_{1}\right)<0$ and $H^{\prime}\left(\omega_{2}\right)>0$ (see lemma 3.2 in [50]).

By Eqs. (3.11) and (3.12), we obtained following equation,

$\cos \omega \tau=\frac{(E-A D) \omega^{2}+(C D-B E)}{D^{2} \omega^{2}+E^{2}}$.

The critical value of time delay is given by

$\tau_{j}^{k}=\frac{1}{\omega_{j}}\left[\arccos \left\{\frac{(E-A D) \omega_{j}^{2}+(C D-B E)}{D^{2} \omega_{j}^{2}+E^{2}}\right\}+2 k \pi\right]$,

where $\mathrm{j}=1,2, \mathrm{k}=0,1,2, \ldots$

Lemma 2 If Hypothesis $\left(\mathrm{H}_{3}\right)$ holds, then Eq. (3.8) has a pair of pure imaginary roots $\pm i \omega_{j}$ and all other roots have nonzero real parts at $\tau=\tau_{j}^{k}(j=1,2, k=0,1,2, \ldots)$.

In addition, we define $\tau_{0}=\min _{j=1,2}\left\{\tau_{j}^{0}\right\}$, i.e., $\tau_{0}$ represents the smallest positive value of $\tau_{j}^{0}, j=1,2$ given by Eq. (3.18) and $\omega_{0}=\omega_{j_{0}}$.

Lemma 3 If Hypothesis $\left(\mathrm{H}_{3}\right)$ holds, then we have the following two transversality conditions:

$\operatorname{sign}\left\{\frac{\mathrm{d} \Re \lambda(\tau)}{\mathrm{d} \tau}\right\}_{\tau=\tau_{1}^{k}}<0$ and $\operatorname{sign}\left\{\frac{\mathrm{d} \Re \lambda(\tau)}{\mathrm{d} \tau}\right\}_{\tau=\tau_{2}^{k}}>0$, where $k=0,1,2 \ldots$

Proof Differentiating Eq. (3.8) w.r.t. $\tau$, we obtained

$\left(\frac{\mathrm{d} \lambda}{\mathrm{d} \tau}\right)^{-1}=\frac{\left(3 \lambda^{2}+2 A \lambda+B\right) \mathrm{e}^{\lambda \tau}}{\lambda\left(D \lambda^{2}+E \lambda\right)}+\frac{(2 D \lambda+E)}{\lambda\left(D \lambda^{2}+E \lambda\right)}-\frac{\tau}{\lambda}$.

Then,

$\left[\Re\left(\frac{\mathrm{d} \lambda}{\mathrm{d} \tau}\right)^{-1}\right]_{\tau=\tau_{j}}=\frac{H^{\prime}\left(\omega_{j}^{2}\right)}{V_{j} \omega_{j}^{2}}$

where

$V_{j}=D^{2} \omega_{j}^{2}+E^{2}$,

where $\mathrm{j}=1,2$.
Therefore, the required transversality condition is obtained if $H^{\prime}\left(\omega_{1}^{2}\right)<0$ and $H^{\prime}\left(\omega_{2}^{2}\right)>0$.

\subsection{Global stability analysis about $E^{*}\left(x^{*}, y^{*}, z^{*}\right)$}

For global stability analysis, we have following theorem:

Theorem 3 If $\min \alpha_{1}, \alpha_{2}, \alpha_{3}>0$, with

$\alpha_{1}=\left\{\left(\frac{r}{K}-\frac{c_{1} y^{*}}{m b_{1} x^{* 2}}-\frac{c_{1} y^{*}}{m_{1} x^{*}}\right)-\frac{2 e_{1} c_{1} y^{*}}{m^{\prime} b_{1} x^{*}}\left(1+\frac{e_{1} c_{1} M^{\prime} \tau}{m^{\prime} b_{1} x^{*}}\right)\right\}$,

$\alpha_{2}=\left\{\frac{c_{1}}{m_{1}}+\frac{e_{1} c_{1}}{M^{\prime} b_{1} x^{*}}-\frac{c_{2} z^{*}}{m^{\prime} b_{2} y^{* 2}}-\frac{c_{2} z^{*}}{m_{2} y^{*}}-\frac{e_{1} c_{1}}{m^{\prime} b_{1} x^{*}}\right.$

$\left.-\frac{e_{1} c_{1} M^{\prime} \tau}{m^{\prime} b_{1} x^{*} y^{*}}\left(\frac{e_{1} c_{1} y^{*}}{M^{\prime} b_{1} x^{*}}+\frac{c_{2} z^{*}}{m^{\prime} b_{2} y^{*}}+\frac{c_{2} z^{*}}{m_{2}}\right)-\frac{2 e_{2} c_{2} z^{*}}{m^{\prime \prime} b_{2} y^{* 2}}\right\}$,

$\alpha_{3}=\left\{-\frac{c_{2}}{m_{2}}\left(1+\frac{e_{1} c_{1} M^{\prime} \tau}{m^{\prime} b_{1} x^{*}}\right)+\frac{e_{2} c_{2}}{M^{\prime \prime} b_{2} y^{*}}-\frac{e_{2} c_{2}}{m^{\prime \prime} b_{2} y^{*}}\right\}$,

where $m<x(t)<M, m^{\prime}<y(t)<M^{\prime}$ and $m^{\prime \prime}<z(t)<M^{\prime \prime}$ for $t>0$, then the interior equilibrium $E^{*}$ of the model system (2.1) is globally asymptotically stable.

Proof A suitable Lyapunov function is constructed for proving the theorem. This function should guarantee the global stability of the positive interior of equilibrium $E^{*}\left(x^{*}, y^{*}, z^{*}\right)$ for system model (2.1). We transform the variables by using the following substitutions:

$x=x^{*} \mathrm{e}^{X(t)}, \quad y=y^{*} \mathrm{e}^{Y(t)}, \quad z=z^{*} \mathrm{e}^{Z(t)}$.

The interior equilibrium $E^{*}$ for trivial equilibrium condition $X(t), Y(t), Z(t)=0$ for all value of $t>0$ is transformed by the above-mentioned coordinates. Reduced model system (2.1) is as follows:

$$
\begin{aligned}
\frac{\mathrm{d} X}{\mathrm{~d} t}= & -\frac{r X^{*}}{K}\left(\mathrm{e}^{X(t)}-1\right) \\
& -\frac{c_{1} y^{*}\left(b_{1} x^{* 2}+m_{1}\right)}{\left(b_{1} x^{2}+m_{1}\right)\left(b_{1} X^{* 2}+m_{1}\right)}\left(\mathrm{e}^{Y(t)}-1\right) \\
& +\frac{b_{1} c_{1} x y^{*} x^{*}}{\left(b_{1} x^{2}+m_{1}\right)\left(b_{1} X^{* 2}+m_{1}\right)}\left(\mathrm{e}^{X(t)}-1\right) \\
& +\frac{b_{1} c_{1} x^{* 2} y^{*}}{\left(b_{1} x^{2}+m_{1}\right)\left(b_{1} X^{* 2}+m_{1}\right)}\left(\mathrm{e}^{X(t)}-1\right),
\end{aligned}
$$




$$
\begin{aligned}
\frac{\mathrm{d} Y}{\mathrm{~d} t}= & -\frac{e_{1} c_{1} x^{*} y^{*}}{y\left(b_{1} x^{* 2}+m_{1}\right)}\left(\mathrm{e}^{Y(t)}-1\right) \\
& +\frac{e_{1} c_{1} x(t-\tau) y^{*}}{y\left(b_{1} x^{2}(t-\tau)+m_{1}\right)}\left(\mathrm{e}^{Y(t-\tau)}-1\right) \\
& +\frac{e_{1} c_{1} x^{*} y^{*}\left(m_{1}-b_{1} x^{*} x(t-\tau)\right)}{y\left(b_{1} x^{* 2}+m_{1}\right)\left(b_{1} x^{2}(t-\tau)+m_{1}\right)} \\
& \times\left(\mathrm{e}^{X(t-\tau)}-1\right) \\
& +\frac{b_{2} c_{2} y y^{*} z^{*}}{\left(b_{2} y^{2}+m_{2}\right)\left(b_{2} y^{* 2}+m_{2}\right)}\left(\mathrm{e}^{Y(t)}-1\right) \\
& +\frac{b_{2} c_{2} y^{* 2} z^{*}}{\left(b_{2} y^{2}+m_{2}\right)\left(b_{2} y^{* 2}+m_{2}\right)}\left(\mathrm{e}^{Y(t)}-1\right) \\
& -\frac{c_{2} z^{*}\left(b_{2} y^{* 2}+m_{2}\right)}{\left(b_{2} y^{2}+m_{2}\right)\left(b_{2} y^{* 2}+m_{2}\right)}\left(\mathrm{e}^{Z(t)}-1\right) \\
\frac{\mathrm{d} Z}{\mathrm{~d} t}= & -\frac{e_{2} c_{2} y^{*} z^{*}}{z\left(b_{2} y^{* 2}+m_{2}\right)}\left(\mathrm{e}^{Z(t)}-1\right) \\
& +\frac{e_{2} c_{2} y z^{*}\left(b_{2} y^{* 2}+m_{2}\right)}{z\left(b_{2} y^{2}+m_{2}\right)\left(b_{2} y^{* 2}+m_{2}\right)}\left(\mathrm{e}^{Z(t)}-1\right) \\
& +\frac{e_{2} c_{2} y^{*} z^{*}\left(m_{2}-b_{2} y y^{*}\right)}{z\left(b_{2} y^{2}+m_{2}\right)\left(b_{2} y^{* 2}+m_{2}\right)} \\
& \times\left(\mathrm{e}^{Y(t)}-1\right)
\end{aligned}
$$

Let $V_{1}(t)=|X(t)|$. Evaluating the upper right derivative of $V_{1}(t)$ along the obtained results of (2.1), we get

$$
\begin{aligned}
D^{+} V_{1}(t) \leq & -\frac{r x^{*}}{K}\left|\mathrm{e}^{X(t)}-1\right| \\
& -\frac{c_{1} y^{*}}{\left(b_{1} x^{2}+m_{1}\right)}\left|\mathrm{e}^{Y(t)}-1\right| \\
& +\frac{b_{1} c_{1} x y^{*} x^{*}}{\left(b_{1} x^{2}+m_{1}\right)\left(b_{1} x^{* 2}+m_{1}\right)}\left|\mathrm{e}^{X(t)}-1\right| \\
& +\frac{b_{1} c_{1} x^{* 2} y^{*}}{\left(b_{1} x^{2}+m_{1}\right)\left(b_{1} x^{* 2}+m_{1}\right)}\left|\mathrm{e}^{X(t)}-1\right|, \\
\leq & -\frac{r x^{*}}{K}\left|\mathrm{e}^{X(t)}-1\right|-\frac{c_{1} y^{*}}{m_{1}}\left|\mathrm{e}^{Y(t)}-1\right| \\
& +\frac{c_{1} y^{*}}{m b_{1} X^{*}}\left|\mathrm{e}^{X(t)}-1\right|+\frac{c_{1} y^{*}}{m_{1}}\left|\mathrm{e}^{X(t)}-1\right| \\
= & -\left(\frac{r x^{*}}{K}-\frac{c_{1} y^{*}}{m b_{1} X^{*}}-\frac{c_{1} y^{*}}{m_{1}}\right)\left|\mathrm{e}^{X(t)}-1\right| \\
& -\frac{c_{1} y^{*}}{m_{1}}\left|\mathrm{e}^{Y(t)}-1\right| .
\end{aligned}
$$

Now, Eq.(3.21) can be rewritten as

$$
\begin{aligned}
\frac{\mathrm{d} Y}{\mathrm{~d} t}= & -\frac{e_{1} c_{1} x^{*} y^{*}}{y\left(b_{1} x^{* 2}+m_{1}\right)}\left(\mathrm{e}^{Y(t)}-1\right) \\
& +\frac{e_{1} c_{1} x^{*} y^{*}\left(m_{1}-b_{1} x^{*} x(t-\tau)\right)}{y\left(b_{1} x^{* 2}+m_{1}\right)\left(b_{1} x^{2}(t-\tau)+m_{1}\right)}\left(\mathrm{e}^{X(t-\tau)}-1\right) \\
& +\frac{b_{2} c_{2} y y^{*} z^{*}}{\left(b_{2} y^{2}+m_{2}\right)\left(b_{2} y^{* 2}+m_{2}\right)}\left(\mathrm{e}^{Y(t)}-1\right) \\
& +\frac{b_{2} c_{2} y^{* 2} z^{*}}{\left(b_{2} y^{2}+m_{2}\right)\left(b_{2} y^{* 2}+m_{2}\right)}\left(\mathrm{e}^{Y(t)}-1\right) \\
& -\frac{c_{2} z^{*}\left(b_{2} y^{* 2}+m_{2}\right)}{\left(b_{2} y^{2}+m_{2}\right)\left(b_{2} y^{* 2}+m_{2}\right)}\left(\mathrm{e}^{Z(t)}-1\right) \\
& +\frac{e_{1} c_{1} x(t-\tau) y^{*}}{y\left(b_{1} x^{2}(t-\tau)+m_{1}\right)}\left(\mathrm{e}^{Y(t)}-1\right) \\
& -\frac{e_{1} c_{1} x(t-\tau) y^{*}}{y\left(b_{1} x^{2}(t-\tau)+m_{1}\right)} \\
& \int_{t-\tau}^{t} \mathrm{e}^{Y(s)}\left\{-\frac{e_{1} c_{1} x^{*} y^{*}}{y\left(b_{1} x^{* 2}+m_{1}\right)}\left(\mathrm{e}^{Y(s)}-1\right)\right. \\
& +\frac{e_{1} c_{1} x^{*} y^{*}\left(m_{1}-b_{1} x^{*} x(t-\tau)\right)}{y\left(b_{1} x^{* 2}+m_{1}\right)\left(b_{1} x^{2}(t-\tau)+m_{1}\right)}\left(\mathrm{e}^{X(s-\tau)}-1\right) \\
& +\frac{b_{2} c_{2} y y^{*} z^{*}}{\left(b_{2} y^{2}+m_{2}\right)\left(b_{2} y^{* 2}+m_{2}\right)} \\
& \times\left(\mathrm{e}^{Y(s)}-1\right)+\frac{b_{2} c_{2} y^{* 2} z^{*}}{\left(b_{2} y^{2}+m_{2}\right)\left(b_{2} y^{* 2}+m_{2}\right)}\left(\mathrm{e}^{Y(s)}-1\right) \\
& -\frac{c_{2} z^{*}\left(b_{2} y^{* 2}+m_{2}\right)}{\left(b_{2} y^{2}+m_{2}\right)\left(b_{2} y^{* 2}+m_{2}\right)} \\
& \left.\times\left(\mathrm{e}^{Z(s)}-1\right)+\frac{e_{1} c_{1} x(t-\tau) y^{*}}{y\left(b_{1} x^{2}(t-\tau)+m_{1}\right)}\left(\mathrm{e}^{Y(s-\tau)}-1\right)\right\} \mathrm{d} s . \\
&
\end{aligned}
$$

In the above equation, we use the following relation

$$
\mathrm{e}^{Y(t-\tau)}=\mathrm{e}^{Y(t)}-\int_{t-\tau}^{t} \mathrm{e}^{Y(s)} \frac{\mathrm{d} Y}{\mathrm{~d} s} \mathrm{~d} s .
$$

Let $V_{2}(t)=|Y(t)|$. Evaluating the upper right derivative of $V_{2}(t)$ along the obtained results of (2.1), following from Eq. (3.21), we get 


$$
\begin{aligned}
D^{+} V_{2}(t) & \leq-\frac{e_{1} c_{1} y^{*}}{M^{\prime} b_{1} x^{*}}\left|\mathrm{e}^{Y(t)}-1\right|+\frac{2 e_{1} c_{1} y^{*}}{m^{\prime} b_{1} x^{*}}\left|\mathrm{e}^{X(t-\tau)}-1\right| \\
& +\frac{c_{2} z^{*}}{m^{\prime} b_{2} y^{*}}\left|\mathrm{e}^{Y(t)}-1\right|+\frac{c_{2} z^{*}}{m_{2}}\left|\mathrm{e}^{Y(t)}-1\right| \\
& +\frac{c_{2} z^{*}}{m_{2}}\left|\mathrm{e}^{Z(t)}-1\right|+\frac{e_{1} c_{1} y^{*}}{m^{\prime} b_{1} x^{*}} \mid \mathrm{e}^{Y(t)} \\
& -1 \mid+\frac{e_{1} c_{1} y^{*}}{m^{\prime} b_{1} x^{*}} \int_{t-\tau}^{t} \mathrm{e}^{Y(s)}\left\{\frac{e_{1} c_{1} y^{*}}{M^{\prime} b_{1} x^{*}}\left|\mathrm{e}^{Y(s)}-1\right|\right. \\
& +\frac{2 e_{1} c_{1} y^{*}}{m^{\prime} b_{1} x^{*}}\left|\mathrm{e}^{X(s-\tau)}-1\right|+\frac{c_{2} z^{*}}{m^{\prime} b_{2} y^{*}}\left|\mathrm{e}^{Y(s)}-1\right| \\
& +\frac{c_{2} z^{*}}{m_{2}}\left|\mathrm{e}^{Y(s)}-1\right|+\frac{c_{2} z^{*}}{m_{2}}\left|\mathrm{e}^{Z(s)}-1\right| \\
& \left.+\frac{e_{1} c_{1} y^{*}}{m^{\prime} b_{1} x^{*}}\left|\mathrm{e}^{Y(s-\tau)}-1\right|\right\} .
\end{aligned}
$$

We find that there exists a $T>0$, such that $y^{*} \mathrm{e}^{Y(t)}<M^{\prime}$ for all $t>T$, and for $t>T+\tau$, we get

$$
\begin{aligned}
D^{+} V_{2}(t) \leq & -\frac{e_{1} c_{1} y^{*}}{M^{\prime} b_{1} x^{*}}\left|\mathrm{e}^{Y(t)}-1\right|+\frac{2 e_{1} c_{1} y^{*}}{m^{\prime} b_{1} x^{*}}\left|\mathrm{e}^{X(t-\tau)}-1\right| \\
& +\frac{c_{2} z^{*}}{m^{\prime} b_{2} y^{*}}\left|\mathrm{e}^{Y(t)}-1\right|+\frac{c_{2} z^{*}}{m_{2}}\left|\mathrm{e}^{Y(t)}-1\right| \\
& +\frac{c_{2} z^{*}}{m_{2}}\left|\mathrm{e}^{Z(t)}-1\right|+\frac{e_{1} c_{1} y^{*}}{m^{\prime} b_{1} x^{*}}\left|\mathrm{e}^{Y(t)}-1\right| \\
& +\frac{e_{1} c_{1} M^{\prime}}{m^{\prime} b_{1} x^{*}} \int_{t-\tau}^{t}\left\{\frac{e_{1} c_{1} y^{*}}{M^{\prime} b_{1} x^{*}}\left|\mathrm{e}^{Y(s)}-1\right|\right. \\
& +\frac{2 e_{1} c_{1} y^{*}}{m^{\prime} b_{1} x^{*}} \\
& \times\left|\mathrm{e}^{X(s-\tau)}-1\right|+\frac{c_{2} z^{*}}{m^{\prime} b_{2} y^{*}}\left|\mathrm{e}^{Y(s)}-1\right| \\
& +\frac{c_{2} z^{*}}{m_{2}}\left|\mathrm{e}^{Y(s)}-1\right|+\frac{c_{2} z^{*}}{m_{2}}\left|\mathrm{e}^{Z(s)}-1\right| \\
& \left.+\frac{e_{1} c_{1} y^{*}}{m^{\prime} b_{1} x^{*}}\left|\mathrm{e}^{Y(s-\tau)}-1\right|\right\} \mathrm{d} s .
\end{aligned}
$$

Again due to structure of (3.27), we consider the following functional

$$
\begin{aligned}
V_{22}(t)= & V_{2}(t)+\frac{e_{1} c_{1} M^{\prime}}{m^{\prime} b_{1} x^{*}} \int_{t-\tau}^{t} \int_{Y}^{t}\left\{\frac{e_{1} c_{1} y^{*}}{M^{\prime} b_{1} x^{*}}\left|\mathrm{e}^{Y(s)}-1\right|\right. \\
& +\frac{2 e_{1} c_{1} y^{*}}{m^{\prime} b_{1} x^{*}}\left|\mathrm{e}^{X(s-\tau)}-1\right| \\
& +\frac{c_{2} z^{*}}{m^{\prime} b_{2} y^{*}}\left|\mathrm{e}^{Y(s)}-1\right|+\frac{c_{2} z^{*}}{m_{2}}\left|\mathrm{e}^{Y(s)}-1\right| \\
& \left.+\frac{c_{2} z^{*}}{m_{2}}\left|\mathrm{e}^{Z(s)}-1\right|+\frac{e_{1} c_{1} y^{*}}{m^{\prime} b_{1} x^{*}}\left|\mathrm{e}^{Y(s-\tau)}-1\right|\right\} d s d Y \\
& +\frac{2 e_{1}^{2} c_{1}^{2} M^{\prime} y^{*} \tau}{m^{\prime 2} b_{1}^{2} x^{* 2}} \int_{t-\tau}^{t}\left|\mathrm{e}^{X(s)}-1\right| \mathrm{d} s \\
& +\frac{e_{1}^{2} c_{1}^{2} M^{\prime} y^{*} \tau}{m^{\prime 2} b_{1}^{2} x^{* 2}} \int_{t-\tau}^{t}\left|\mathrm{e}^{Y(s)}-1\right| \mathrm{d} s \\
& +\frac{2 e_{1} c_{1} y^{*}}{m^{\prime} b_{1} x^{*}} \int_{t-\tau}^{t}\left|\mathrm{e}^{X(s)}-1\right| \mathrm{d} s,
\end{aligned}
$$

whose upper right derivative along the solutions of the system (2.1) is given by

$$
\begin{aligned}
& D^{+} V_{22}(t) \\
& =D^{+} V_{2}+\frac{e_{1} c_{1} M^{\prime} \tau}{m^{\prime} b_{1} x^{*}}\left\{\frac{e_{1} c_{1} y^{*}}{M^{\prime} b_{1} x^{*}}\left|\mathrm{e}^{Y(t)}-1\right|\right. \\
& +\frac{2 e_{1} c_{1} y^{*}}{m^{\prime} b_{1} x^{*}}\left|\mathrm{e}^{X(t-\tau)}-1\right|+\frac{c_{2} z^{*}}{m^{\prime} b_{2} y^{*}}\left|\mathrm{e}^{Y(t)}-1\right| \\
& +\frac{c_{2} z^{*}}{m_{2}}\left|\mathrm{e}^{Y(t)}-1\right|+\frac{c_{2} z^{*}}{m_{2}}\left|\mathrm{e}^{Z(t)}-1\right| \\
& \left.+\frac{e_{1} c_{1} y^{*}}{m^{\prime} b_{1} x^{*}}\left|\mathrm{e}^{\gamma(t-\tau)}-1\right|\right\}-\frac{e_{1} c_{1} M^{\prime}}{m^{\prime} b_{1} x^{*}} \\
& \times \int_{t-\tau}^{t}\left\{\frac{e_{1} c_{1} y^{*}}{M^{\prime} b_{1} X^{*}}\left|\mathrm{e}^{Y(s)}-1\right|+\frac{2 e_{1} c_{1} y^{*}}{m^{\prime} b_{1} X^{*}}\left|\mathrm{e}^{X(s-\tau)}-1\right|\right. \\
& +\frac{c_{2} z^{*}}{m^{\prime} b_{2} y^{*}}\left|\mathrm{e}^{Y(s)}-1\right|+\frac{c_{2} z^{*}}{m_{2}}\left|\mathrm{e}^{Y(s)}-1\right| \\
& \left.+\frac{c_{2} z^{*}}{m_{2}}\left|\mathrm{e}^{Z(s)}-1\right|+\frac{e_{1} c_{1} y^{*}}{m^{\prime} b_{1} x^{*}}\left|\mathrm{e}^{Y(s-\tau)}-1\right|\right\} \\
& +\frac{e_{1}^{2} c_{1}^{2} M^{\prime} y^{*} \tau}{m^{\prime 2} b_{1}^{2} x^{* 2}}\left[\left|\mathrm{e}^{X(t)}-1\right|-\left|\mathrm{e}^{X(t-\tau)}-1\right|\right] \\
& +\left\{\frac{e_{1}^{2} c_{1}^{2} M^{\prime} y^{*} \tau}{m^{\prime 2} b_{1}^{2} x^{* 2}}\left[\left|\mathrm{e}^{X(t)}-1\right|-\left|\mathrm{e}^{\gamma(t)}-1\right|\right]\right. \\
& \left.-\frac{e_{1}^{2} c_{1}^{2} M^{\prime} y^{*} \tau}{m^{\prime 2} b_{1}^{2} x^{* 2}}\left[\left|\mathrm{e}^{X(t-\tau)}-1\right|-\left|\mathrm{e}^{Y(t-\tau)}-1\right|\right]\right\} \\
& +\frac{2 e_{1} c_{1} y^{*}}{m^{\prime} b_{1} x^{*}}\left[\left|e^{X(t)}-1\right|-\left|\mathrm{e}^{X(t-\tau)}-1\right|\right],
\end{aligned}
$$

which implies 


$$
\begin{aligned}
& D^{+} V_{22}(t) \\
& \leq-\frac{e_{1} c_{1} y^{*}}{M^{\prime} b_{1} x^{*}}\left|e^{Y(t)}-1\right|+\frac{c_{2} z^{*}}{m^{\prime} b_{2} y^{*}}\left|e^{Y(t)-1}\right| \\
&+\frac{c_{2} z^{*}}{m_{2}}\left|\mathrm{e}^{Y(t)}-1\right|+\frac{e_{1} c_{1} y^{*}}{m^{\prime} b_{1} x^{*}}\left|\mathrm{e}^{Y(t)}-1\right| \\
&+\frac{c_{2} z^{*}}{m_{2}}\left|\mathrm{e}^{Z(t)}-1\right|+\frac{e_{1} c_{1} M^{\prime} \tau}{m^{\prime} b_{1} x^{*}}\left\{\frac{e_{1} c_{1} y^{*}}{M^{\prime} b_{1} x^{*}}\left|\mathrm{e}^{Y(t)}-1\right|\right. \\
&+\frac{c_{2} z^{*}}{m^{\prime} b_{2} y^{*}}\left|\mathrm{e}^{Y(t)}-1\right|+\frac{c_{2} z^{*}}{m_{2}}\left|\mathrm{e}^{Y(t)}-1\right| \\
&\left.+\frac{c_{2} z^{*}}{m_{2}}\left|\mathrm{e}^{Z(t)}-1\right|\right\}+\frac{2 e_{1}^{2} c_{1}^{2} M^{\prime} y^{*} \tau}{m^{\prime 2} b_{1}^{2} x^{* 2}}\left|\mathrm{e}^{X(t)}-1\right| \\
&= \frac{2 e_{1} c_{1} y^{*}}{m^{\prime} b_{1} x^{*}}\left(1+\frac{e_{1} c_{1} M^{\prime} \tau}{m^{\prime} b_{1} x^{*}}\right)\left|\mathrm{e}^{X(t)}-1\right| \\
&+\left[-\frac{e_{1} c_{1} y^{*}}{M^{\prime} b_{1} x^{*}}+\frac{c_{2} z^{*}}{m^{\prime} b_{2} y^{*}}\right. \\
&+\frac{c_{2} z^{*}}{m_{2}}+\frac{e_{1} c_{1} y^{*}}{m^{\prime} b_{1} x^{*}}+\frac{e_{1} c_{1} M^{\prime} \tau}{m^{\prime} b_{1} x^{*}}\left\{\frac{e_{1} c_{1} y^{*}}{M^{\prime} b_{1} x^{*}}+\frac{c_{2} z^{*}}{m^{\prime} b_{2} y^{*}}\right. \\
&\left.\left.+\frac{c_{2} z^{*}}{m_{2}}\right\}\right]+\frac{c_{2} z^{*}}{m_{2}}\left(1+\frac{e_{1} c_{1} M^{\prime} \tau}{m^{\prime} b_{1} x^{*}}\right)\left|\mathrm{e}^{Z(1)}-1\right| .
\end{aligned}
$$

Again, Let $V_{3}(t)=|Z(t)|$. Evaluating the upper right derivative of $V_{3}(t)$ along the obtained results of (2.1), we get

$$
\begin{aligned}
D^{+} V_{3}(t) \leq & -\frac{e_{2} c_{2} z^{*}}{b_{2} y^{*} z}\left|\mathrm{e}^{Z(t)}-1\right|+\frac{e_{2} c_{2} z^{*}}{b_{2} y^{*} z}\left|\mathrm{e}^{Z(t)}-1\right| \\
& +\frac{2 e_{2} c_{2} z^{*}}{b_{2} y^{*} z}\left|\mathrm{e}^{Y(t)}-1\right| \\
\leq & -\frac{e_{2} c_{2} z^{*}}{b_{2} y^{*} M^{\prime \prime}}\left|\mathrm{e}^{Z(t)}-1\right|+\frac{e_{2} c_{2} z^{*}}{b_{2} y^{*} m^{\prime \prime}}\left|\mathrm{e}^{Z(t)}-1\right| \\
& +\frac{2 e_{2} c_{2} z^{*}}{b_{2} y^{*} m^{\prime \prime}}\left|\mathrm{e}^{Y(t)}-1\right| .
\end{aligned}
$$

We define a Lyapunov functional $V(t)$ as

$V(t)=V_{1}(t)+V_{22}(t)+V_{3}(t)>|X(t)|+|Y(t)|+|Z(t)|$.

Evaluating the upper right derivative of $V(t)$ along the obtained results of system (2.1), and by using Eqs. (3.23), (3.29) and (3.30), we get
$D^{+} V(t)$

$$
\begin{aligned}
= & D^{+} V_{1}(t)+D^{+} V_{22}(t)+D^{+} V_{3}(t) \\
\leq & \left\{-\left(\frac{r}{K}-\frac{c_{1} y^{*}}{m b_{1} x^{* 2}}-\frac{c_{1} y^{*}}{m_{1} x^{*}}\right)\right. \\
& \left.+\frac{2 e_{1} c_{1} y^{*}}{m^{\prime} b_{1} x^{*}}\left(1+\frac{e_{1} c_{1} M^{\prime} \tau}{m^{\prime} b_{1} x^{*}}\right)\right\}\left|\mathrm{e}^{X(t)}-1\right|+\left\{-\frac{c_{1}}{m_{1}}\right. \\
& -\frac{e_{1} c_{1}}{M^{\prime} b_{1} X^{*}}-\frac{c_{2} z^{*}}{m^{\prime} b_{2} y^{* 2}}-\frac{c_{2} z^{*}}{m_{2} y^{*}}-\frac{e_{1} c_{1}}{m^{\prime} b_{1} x^{*}} \\
& -\frac{e_{1} c_{1} M^{\prime} \tau}{m^{\prime} b_{1} X^{*} y^{*}}\left(\frac{e_{1} c_{1} y^{*}}{M^{\prime} b_{1} X^{*}}+\frac{c_{2} z^{*}}{m^{\prime} b_{2} y^{*}}+\frac{c_{2} z^{*}}{m_{2}}\right) \\
& \left.+\frac{2 e_{2} c_{2} z^{*}}{m^{\prime \prime} b_{2} y^{* 2}}\right\}\left|\mathrm{e}^{Y(t)}-1\right|+\left\{\frac{c_{2}}{m_{2}}\left(1+\frac{e_{1} c_{1} M^{\prime} \tau}{m^{\prime} b_{1} X^{*}}\right)\right. \\
& \left.-\frac{e_{2} c_{2}}{M^{\prime \prime} b_{2} y^{*}}+\frac{e_{2} c_{2}}{m^{\prime \prime} b_{2} y^{*}}\right\}\left|\mathrm{e}^{Z(t)}-1\right| \\
\leq & -\alpha_{1} X^{*}\left|\mathrm{e}^{X(t)}-1\right|-\alpha_{2} Y^{*}\left|\mathrm{e}^{Y(t)}-1\right| \\
& -\alpha_{3} Z^{*}\left|\mathrm{e}^{Z(t)}-1\right|,
\end{aligned}
$$

where

$$
\begin{aligned}
\alpha_{1}= & \left\{\left(\frac{r}{K}-\frac{c_{1} y^{*}}{m b_{1} x^{* 2}}-\frac{c_{1} y^{*}}{m_{1} x^{*}}\right)\right. \\
& \left.-\frac{2 e_{1} c_{1} y^{*}}{m^{\prime} b_{1} x^{*}}\left(1+\frac{e_{1} c_{1} M^{\prime} \tau}{m^{\prime} b_{1} x^{*}}\right)\right\}>0, \\
\alpha_{2}= & \frac{c_{1}}{m_{1}}+\frac{e_{1} c_{1}}{M^{\prime} b_{1} x^{*}}-\frac{c_{2} z^{*}}{m^{\prime} b_{2} y^{* 2}}-\frac{c_{2} z^{*}}{m_{2} y^{*}}-\frac{e_{1} c_{1}}{m^{\prime} b_{1} x^{*}} \\
& -\frac{e_{1} c_{1} M^{\prime} \tau}{m^{\prime} b_{1} x^{*} y^{*}}\left(\frac{e_{1} c_{1} y^{*}}{M^{\prime} b_{1} x^{*}}+\frac{c_{2} z^{*}}{m^{\prime} b_{2} y^{*}}+\frac{c_{2} z^{*}}{m_{2}}\right) \\
& \left.-\frac{2 e_{2} c_{2} z^{*}}{m^{\prime \prime} b_{2} y^{* 2}}\right\}>0, \\
\alpha_{3}= & \left\{-\frac{c_{2}}{m_{2}}\left(1+\frac{e_{1} c_{1} M^{\prime} \tau}{m^{\prime} b_{1} x^{*}}\right)+\frac{e_{2} c_{2}}{M^{\prime \prime} b_{2} y^{*}}-\frac{e_{2} c_{2}}{m^{\prime \prime} b_{2} y^{*}}\right\}>0 .
\end{aligned}
$$

Since the model system (2.1) is positive invariant, for all $t>T^{*}$, we have

$$
\begin{aligned}
x^{*}\left|\mathrm{e}^{X(t)}\right|=x(t)>m, \quad y^{*}\left|\mathrm{e}^{Y(t)}\right|=y(t)>m^{\prime}, \\
z^{*}\left|\mathrm{e}^{Z(t)}\right|=z(t)>m^{\prime \prime} .
\end{aligned}
$$

Using mean value theorem, we have

$$
\begin{aligned}
& x^{*}\left|\mathrm{e}^{X(t)}-1\right|=x^{*} \mathrm{e}^{\theta_{1}(t)}|X(t)|>m|X(t)|, \\
& y^{*}\left|\mathrm{e}^{Y(t)}-1\right|=y^{*} \mathrm{e}^{\theta_{1}(t)}|Y(t)|>m^{\prime}|Y(t)|, \\
& z^{*}\left|\mathrm{e}^{Z(t)}-1\right|=z^{*} \mathrm{e}^{\theta_{1}(t)}|Z(t)|>m^{\prime \prime}|Z(t)|,
\end{aligned}
$$


where $x^{*} \mathrm{e}^{\theta_{1}(t)}$ lies between $x^{*}$ and $x(t), y^{*} \mathrm{e}^{\theta_{1}(t)}$ lies between $y^{*}$ and $y(t)$ and $z^{*} \mathrm{e}^{\theta_{1}(t)}$ lies between $z^{*}$ and $z(t)$. Therefore,

$$
\begin{aligned}
D^{+} V(t) & \leq-\alpha_{1} m|X(t)|-\alpha_{2} m^{\prime}|Y(t)|-\alpha_{3} m^{\prime \prime}|Z(t)| \\
& \leq-\gamma(|X(t)|+|Y(t)|+|Z(t)|)
\end{aligned}
$$

where $\gamma=\min \left\{\alpha_{1} m, \alpha_{2} m^{\prime}, \alpha_{3} m^{\prime \prime}\right\}$.

Noting that $V(t)>|X(t)|+|Y(t)|+|Z(t)|$. Hence, by using Eq. (3.33) and Lyapunov's direct method, we can conclude that the zero solution of the reduced system (3.20)-(3.22) is globally asymptotically stable. Therefore, the positive equilibrium of the original model system (2.1) is globally asymptotically stable.

\subsection{Stability and direction of the Hopf bifurcation}

This section is based on the numerical analysis of Hopf bifurcation theory by using center manifold theorem and

$$
\begin{aligned}
& J=\left(\begin{array}{ccc}
-\frac{r x^{*}}{K}+\frac{2 b_{1} c_{1} x^{* 2} y^{*}}{\gamma_{1}^{2}} & -\frac{c_{1} x^{*}}{\gamma_{1}} & 0 \\
0 & -d_{1}+\frac{\delta_{4} c_{2} z^{*}}{\gamma_{2}^{2}} & -\frac{c_{2} y^{*}}{\gamma_{2}} \\
0 & -\frac{\delta_{4} e_{2} c_{2} z^{*}}{\gamma_{2}^{2}} & 0
\end{array}\right), \\
& J^{\prime}=\left(\begin{array}{ccc}
0 & 0 & 0 \\
-\frac{\delta_{2} e_{1} c_{1} y^{*}}{\gamma_{1}^{2}} & \frac{e_{1} c_{1} x^{*}}{\gamma_{1}} & 0 \\
0 & 0 & 0
\end{array}\right),
\end{aligned}
$$

and

$$
=\left(\tau_{0}+\mu\right)\left(\begin{array}{c}
\left(-\frac{r}{K}-\frac{\delta_{1} b_{1} c_{1} x^{*} y^{*}}{\gamma_{1}^{3}}\right) \phi_{1}^{2}(0)+\frac{\delta_{2} c_{1}}{\gamma_{1}^{2}} \phi_{1}(0) \phi_{2}(0) \\
\frac{\delta_{1} e_{1} b_{1} c_{1} x^{*} y^{*}}{\gamma_{1}^{3}} \phi_{1}^{2}(-1)-\frac{\delta_{2} e_{1} c_{1}}{\gamma_{1}^{2}} \phi_{1}(-1) \phi_{2}(-1)+\frac{\delta_{4} c_{2}}{\gamma_{2}^{2}} \phi_{2}(0) \phi_{3}(0)-\frac{\delta_{3} b_{2} c_{2} y^{*} z^{*}}{\gamma_{2}^{3}} \phi_{2}^{2}(0) \\
\frac{\delta_{3} e_{2} b_{2} c_{2} y^{*} z^{*}}{\gamma_{2}^{3}} \phi_{2}^{2}(0)-\frac{\delta_{4} e_{2} c_{2}}{\gamma_{2}^{2}} \phi_{2}(0) \phi_{3}(0)
\end{array}\right),
$$

normal form theory [51], where the critical value of time delay is denoted by $\tau=\tau_{0}$ at which Eq. (3.8) has a pair of purely imaginary roots $\pm i \omega_{0}$ and system undergoes a Hopf bifurcation at equilibrium $E^{*}$.

Let $x_{1}=x-x^{*}, x_{2}=y-y^{*}, x_{3}=z-z^{*}, \mu=\tau-\tau_{0}$ where $\mu \in \mathfrak{R}$. Rescaling the time by $t \longrightarrow \frac{t}{\tau}$, the system (2.1) can be written into the following continuous realvalued functions $C=\left([-1,0], \Re^{3}\right)$ as

$\dot{x}(t)=L_{\mu}\left(x_{t}\right)+f\left(\mu, x_{t}\right)$,

where $x(t)=\left(x_{1}(t), x_{2}(t), x_{3}(t)\right)^{T} \in \Re^{3}, x_{t}(\theta)=x(t+\theta), \theta \in[-1,0]$ and $L_{\mu}: C \rightarrow \mathfrak{R}^{3}, f: \mathfrak{R} \times C \rightarrow \mathfrak{R}^{3}$ are given, respectively,

$L_{\mu}(\phi)=\left(\tau_{0}+\mu\right)\left[J \phi(0)+J^{\prime} \phi(-1)\right]$,

such that where $\delta_{1}=\left(b_{1} x^{* 2}-3 m_{1}\right), \delta_{2}=\left(b_{1} x^{* 2}-m_{1}\right), \delta_{3}=\left(b_{2} y^{* 2}-3 m_{2}\right)$, $\delta_{4}=\left(b_{2} y^{* 2}-m_{2}\right), \gamma_{1}=\left(b_{1} x^{* 2}+m_{1}\right) \gamma_{2}=\left(b_{2} y^{* 2}+m_{2}\right)$ and $\phi(\theta)=\left(\phi_{1}(\theta), \phi_{2}(\theta), \phi_{3}(\theta)\right)^{T} \in C\left([-1,0], \mathfrak{R}^{3}\right)$.

By the Riesz representation theorem, there exists a function $\eta(\theta, \mu)$ of bounded variation for $\theta \in[-1,0]$, such that

$L_{\mu} \phi=\int_{-1}^{0} \mathrm{~d} \eta(\theta, \mu) \phi(\theta), \quad$ for $\quad \phi \in C$.

In fact, we can take

$\eta(\theta, \mu)=\left(\tau_{0}+\mu\right)\left[J \delta(\theta)+J^{\prime} \delta(\theta+1)\right]$,

where $\delta(\theta)$ is the Dirac delta function.

For $\phi \in C\left([-1,0], \mathfrak{R}^{3}\right)$, define

$A(\mu) \phi= \begin{cases}\frac{\mathrm{d} \phi(\theta)}{\mathrm{d} \theta}, & \theta \in[-1,0), \\ \int_{-1}^{0} \phi(s) \mathrm{d} \eta(s, \mu), & \theta=0,\end{cases}$ 
and

$R(\mu) \phi= \begin{cases}0, & \theta \in[-1,0), \\ f(\mu, \phi), & \theta=0 .\end{cases}$

Then, Eq. (3.34) equivalent to the following equation,

$\dot{x}_{t}=A(\mu) x_{t}+R(\mu) x_{t}$

where $x_{t}(\theta)=x(t+\theta)$ for $\theta \in[-1,0]$.

For $\psi \in C^{1}\left([0,1],\left(\Re^{3}\right)^{*}\right)$, we define the adjoint of $A$ as

$A^{*} \psi(s)= \begin{cases}-\frac{\mathrm{d} \psi(s)}{\mathrm{d} s} & s \in(0,1], \\ \int_{-1}^{0} \psi(-t) \mathrm{d} \eta^{T}(t, 0), & s=0,\end{cases}$

and a bilinear inner product is given by

$\langle\psi(s), \phi(\theta)\rangle=\bar{\psi}(0) \phi(0)-\int_{-1}^{0} \int_{0}^{\theta} \bar{\psi}(\xi-\theta) \mathrm{d} \eta(\theta) \phi(\xi) \mathrm{d} \xi$,

where $\eta(\theta)=\eta(\theta, 0)$. Then, $A(0)$ and $A^{*}$ are adjoint operators.

For further calculation, we assume that $i \omega_{0} \tau_{0}$ and $-i \omega_{0} \tau_{0}$ are eigenvalues of $A(0)$ and $A^{*}$, respectively. Now, let $q(\theta)=\left(1, \alpha_{2}, \alpha_{3}\right)^{T} \mathrm{e}^{i \omega_{0} \tau_{0} \theta}$ and $q^{*}(s)=N\left(1, \alpha_{2}^{*}, \alpha_{3}^{*}\right) \mathrm{e}^{i \omega_{0} \tau_{0} s}$ are the eigenvector of $A(0)$ and $A^{*}(0)$ corresponding to $+i \omega_{0} \tau_{0}$ and $-i \omega_{0} \tau_{0}$, respectively.

Then,

$A q(\theta)=i \omega_{0} \tau_{0} q(\theta)$,

for $\theta=0$, we obtained

$\tau_{0}\left(\begin{array}{ccc}i \omega_{0}+\frac{r x^{*}}{K}-\frac{2 b_{1} c_{1} x^{* 2} y^{*}}{\gamma_{1}^{2}} & \frac{c_{1} x^{*}}{\gamma_{1}} & 0 \\ \mathrm{e}^{-i \omega_{0} \tau_{0}} \frac{\delta_{2} e_{1} c_{1} y^{*}}{\gamma_{1}^{2}} & i \omega_{0}-\frac{\delta_{4} c_{2} z^{*}}{\gamma_{2}^{2}}+d_{1}-\mathrm{e}^{-i \omega_{0} \tau_{0}} \frac{e_{1} c_{1} x^{*}}{\gamma_{1}} & \frac{c_{2} y^{*}}{\gamma_{2}} \\ 0 & \frac{\delta_{4} e_{2} c_{2} z^{*}}{\gamma_{2}^{2}} & i \omega_{0}\end{array}\right) q(0)=\left(\begin{array}{l}0 \\ 0 \\ 0\end{array}\right)$.
Solving the system of equations, we get

$\alpha_{2}=\frac{2 b_{1} x^{*} y^{*}}{\gamma_{1}}-\frac{r \gamma_{1}}{K c_{1}}-\frac{i \omega_{0} \gamma_{1}}{c_{1} x^{*}}$

and

$\alpha_{3}=-\frac{\alpha_{2} \delta_{4} e_{2} c_{2} z^{*}}{i \omega_{0} \gamma_{2}^{2}}$.

Similarly, let

$A^{*} q^{*}(s)=-i \omega_{0} \tau_{0} q^{*}(s)$,

where

$\alpha_{2}^{*}=\frac{2 b_{1} x^{* 2}}{\mathrm{e}^{-i \omega_{0} \tau_{0}} \delta_{2} e_{1}}-\frac{r x^{*} \gamma_{1}^{2}}{\mathrm{e}^{-i \omega_{0} \tau_{0}} \delta_{2} K e_{1} c_{1} y^{*}}+\frac{i \omega_{0} \gamma_{1}^{2}}{\mathrm{e}^{-i \omega_{0} \tau_{0}} \delta_{2} e_{1} c_{1} y^{*}}$,

and

$\alpha_{3}^{*}=\frac{c_{2} y^{*} \alpha_{2}^{*}}{i \omega_{0} \gamma_{2}}$.

Under the normalization condition $\left\langle q^{*}(s), q(\theta)\right\rangle=1$, we have

$N=\frac{1}{\bar{D}}$

where

$D=1+\alpha_{2} \bar{\alpha}_{2}^{*}+\alpha_{3} \bar{\alpha}_{3}^{*}+\tau_{0} \bar{\alpha}_{2}^{*}\left[-\frac{\delta_{2} e_{1} c_{1} y^{*}}{\gamma_{1}^{2}}+\alpha_{2} \frac{e_{1} c_{1} x^{*}}{\gamma_{1}}\right] \mathrm{e}^{-i \omega_{0} \tau_{0}}$.

Now in the remainder section, following in the same manner as given in [51], we obtained 
$g(z, \bar{z})$

$$
=\tau_{0} \bar{N}\left(1, \bar{\alpha}_{2}^{*}, \bar{\alpha}_{3}^{*}\right)\left(\begin{array}{c}
\left(-\frac{r}{K}-\frac{\delta_{1} b_{1} c_{1} x^{*} y^{*}}{\gamma_{1}^{3}}\right) \phi_{1}^{2}(0)+\frac{\delta_{2} c_{1}}{\gamma_{1}^{2}} \phi_{1}(0) \phi_{2}(0) \\
\delta_{1} e_{1} b_{1} c_{1} x^{*} y^{*} \\
\gamma_{1}^{3}(-1)-\frac{\delta_{2} e_{1} c_{1}}{\gamma_{1}^{2}} \phi_{1}(-1) \phi_{2}(-1)+\frac{\delta_{4} c_{2}}{\gamma_{2}^{2}} \phi_{2}(0) \phi_{3}(0)-\frac{\delta_{3} b_{2} c_{2} y^{*} z^{*}}{\gamma_{2}^{3}} \phi_{2}^{2}(0) \\
\frac{\delta_{3} e_{2} b_{2} c_{2} y^{*} z^{*}}{\gamma_{2}^{3}} \phi_{2}^{2}(0)-\frac{\delta_{4} e_{2} c_{2}}{\gamma_{2}^{2}} \phi_{2}(0) \phi_{3}(0)
\end{array}\right)
$$

such that

$$
\begin{aligned}
\phi_{1}(0)= & z+\bar{z}+W_{20}^{(1)}(0) \frac{z^{2}}{2}+W_{11}^{(1)}(0) z \bar{z}+W_{02}^{(1)}(0) \frac{\bar{z}^{2}}{2} \\
& +\ldots, \\
\phi_{2}(0)= & \alpha_{2} z+\bar{\alpha}_{2} \bar{z}+W_{20}^{(2)}(0) \frac{z^{2}}{2}+W_{11}^{(2)}(0) z \bar{z}+W_{02}^{(2)}(0) \frac{\bar{z}^{2}}{2} \\
& +\ldots, \\
\phi_{3}(0)= & \alpha_{3} z+\bar{\alpha}_{3} \bar{z}+W_{20}^{(3)}(0) \frac{z^{2}}{2}+W_{11}^{(3)}(0) z \bar{z}+W_{02}^{(3)}(0) \frac{\bar{z}^{2}}{2} \\
& +\ldots, \\
\phi_{1}(-1)= & z \mathrm{e}^{-i \omega_{0} \tau_{0}}+\bar{z} \mathrm{e}^{i \omega_{0} \tau_{0}}+W_{20}^{(1)}(-1) \frac{z^{2}}{2}+W_{11}^{(1)}(-1) z \bar{z} \\
& +W_{02}^{(1)}(-1) \frac{\bar{z}^{2}}{2}+\ldots, \\
\phi_{2}(-1)= & \alpha_{2} z \mathrm{e}^{-i \omega_{0} \tau_{0}}+\bar{\alpha}_{2} \bar{z} \mathrm{e}^{i \omega_{0} \tau_{0}}+W_{20}^{(2)}(-1) \frac{z^{2}}{2} \\
& +W_{11}^{(2)}(-1) z \bar{z}+W_{02}^{(2)}(-1) \frac{\bar{z}^{2}}{2}+\ldots
\end{aligned}
$$

From Eq. (3.47), we have the following quantities:

$$
\begin{aligned}
g_{20}= & 2 \tau_{0} \bar{N}\left[\left(\left(-\frac{r}{K}-\frac{\delta_{1} b_{1} c_{1} x^{*} y^{*}}{\gamma_{1}^{3}}\right)\right.\right. \\
& \left.+\frac{\delta_{1} e_{1} b_{1} c_{1} x^{*} y^{*}}{\gamma_{1}^{3}} \bar{\alpha}_{2}^{*} \mathrm{e}^{-2 i \omega_{0} \tau_{0}}\right) \\
& +\alpha_{2}\left(\frac{\delta_{2} c_{1}}{\gamma_{1}^{2}}-\frac{\delta_{2} e_{1} c_{1}}{\gamma_{1}^{2}} \bar{\alpha}_{2}^{*} \mathrm{e}^{-2 i \omega_{0} \tau_{0}}\right) \\
& +\alpha_{2} \alpha_{3}\left(\frac{\delta_{4} c_{2}}{\gamma_{2}^{2}} \bar{\alpha}_{2}^{*}-\frac{\delta_{4} e_{2} c_{2}}{\gamma_{2}^{2}} \bar{\alpha}_{3}^{*}\right) \\
& \left.+\alpha_{2}^{2}\left(-\frac{\delta_{3} b_{2} c_{2} y^{*} z^{*}}{\gamma_{2}^{3}} \bar{\alpha}_{2}^{*}+\frac{\delta_{3} e_{2} b_{2} c_{2} y^{*} z^{*}}{\gamma_{2}^{3}} \bar{\alpha}_{3}^{*}\right)\right]
\end{aligned}
$$

$$
\begin{array}{rl}
g_{11}= & 2 \tau_{0} \bar{N}\left[\left(-\frac{r}{K}-\frac{\delta_{1} b_{1} c_{1} x^{*} y^{*}}{\gamma_{1}^{3}}\right)+\frac{\delta_{1} e_{1} b_{1} c_{1} x^{*} y^{*}}{\gamma_{1}^{3}} \bar{\alpha}_{2}^{*}\right) \\
& +\operatorname{Re}\left(\alpha_{2}\right)\left(\frac{\delta_{2} c_{1}}{\gamma_{1}^{2}}-\frac{\delta_{2} e_{1} c_{1}}{\gamma_{1}^{2}} \bar{\alpha}_{2}^{*}\right)+\operatorname{Re}\left(\alpha_{2} \bar{\alpha}_{3}\right)\left(\frac{\delta_{4} c_{2}}{\gamma_{2}^{2}} \bar{\alpha}_{2}^{*}-\frac{\delta_{4} e_{2} c_{2}}{\gamma_{2}^{2}} \bar{\alpha}_{3}^{*}\right) \\
& \left.+\alpha_{2} \bar{\alpha}_{2}\left(-\frac{\delta_{3} b_{2} c_{2} y^{*} z^{*}}{\gamma_{2}^{3}} \bar{\alpha}_{2}^{*}+\frac{\delta_{3} e_{2} b_{2} c_{2} y^{*} z^{*}}{\gamma_{2}^{3}} \bar{\alpha}_{3}^{*}\right)\right] \\
g_{02}= & 2 \tau_{0} \bar{N}\left[\left(-\frac{r}{K}-\frac{\delta_{1} b_{1} c_{1} x^{*} y^{*}}{\gamma_{1}^{3}}\right)+\frac{\delta_{1} e_{1} b_{1} c_{1} x^{*} y^{*}}{\gamma_{1}^{3}} \bar{\alpha}_{2}^{*} \mathrm{e}^{2 i \omega_{0} \tau_{0}}\right) \\
& +\bar{\alpha}_{2}\left(\frac{\delta_{2} c_{1}}{\gamma_{1}^{2}}-\frac{\delta_{2} e_{1} c_{1}}{\gamma_{1}^{2}} \bar{\alpha}_{2}^{*} \mathrm{e}^{2 i \omega_{0} \tau_{0}}\right)+\bar{\alpha}_{2} \bar{\alpha}_{3}\left(\frac{\delta_{4} c_{2}}{\gamma_{2}^{2}} \bar{\alpha}_{2}^{*}-\frac{\delta_{4} e_{2} c_{2}}{\gamma_{2}^{2}} \bar{\alpha}_{3}^{*}\right) \\
& \left.+\bar{\alpha}_{2}^{2}\left(-\frac{\delta_{3} b_{2} c_{2} y^{*} z^{*}}{\gamma_{2}^{3}} \bar{\alpha}_{2}^{*}+\frac{\delta_{3} e_{2} b_{2} c_{2} y^{*} z^{*}}{\gamma_{2}^{3}} \bar{\alpha}_{3}^{*}\right)\right] \\
& \left.\left.+4 \alpha_{2} W_{11}^{(2)}(0)\right)\right] \\
g_{21} & 2 \tau_{0} \bar{N}\left[\left(-\frac{r}{K}-\frac{\delta_{1} b_{1} c_{1} x^{*} y^{*}}{\gamma_{1}^{3}}\right)\left(2 W_{20}^{(1)}(0)+4 W_{11}^{(1)}(0)\right)\right. \\
& \left.+2 \alpha_{3} W_{11}^{(2)}(0)+\bar{\alpha}_{2} W_{20}^{(3)}(0)+2 \alpha_{2} W_{11}^{(3)}(0)\right) \\
& +\frac{\delta_{1} e_{1} b_{1} c_{1} x^{*} y^{*}}{\gamma_{1}^{3}} \bar{\alpha}_{2}^{*}\left(2 \mathrm{e}^{i \omega_{0} \tau_{0}} W_{20}^{(1)}(-1)\right. \\
& \left.+2 \mathrm{e}^{-i \omega_{0} \tau_{0}} W_{11}^{(2)}(-1)\right)+\left(\frac{\delta_{4} c_{2}}{\gamma_{2}^{2}} \bar{\alpha}_{2}^{*}-\frac{\delta_{4} e_{2} c_{2}}{\gamma_{2}^{2}} \bar{\alpha}_{3}^{*}\right)\left(\bar{\alpha}_{3} W_{20}^{(2)}(0)\right. \\
& \left.+4 \mathrm{e}^{-i \omega_{0} \tau_{0}} W_{11}^{(1)}(-1)\right)+\frac{\delta_{2} c_{1}}{\gamma_{1}^{2}}\left(\bar{\alpha}_{2} W_{20}^{(1)}(0)+2 \alpha_{2} W_{11}^{(1)}(0)\right. \\
& \left.+W_{20}^{(2)}(0)+2 W_{11}^{(2)}(0)\right)-\frac{\delta_{2} e_{1} c_{1}}{\gamma_{1}^{2}} \bar{\alpha}_{2}^{*}\left(\bar{\alpha}_{2} \mathrm{e}^{i \omega_{0} \tau_{0}} W_{20}^{(1)}(-1)\right. \\
& +2 \alpha_{2} \mathrm{e}^{-i \omega_{0} \tau_{0}} W_{11}^{(1)}(-1)+\mathrm{e}^{i \omega_{0} \tau_{0}} W_{20}^{(2)}(-1) \\
& \left(2 \bar{\alpha}_{2} W_{20}^{(2)}(0)\right. \\
& \left.-\frac{\delta_{3} e_{2} b_{2} c_{2} y^{*} z^{*}}{\gamma_{2}^{*}} \bar{\alpha}_{3}^{*}\right) \\
& \\
& \\
&
\end{array}
$$

We denote $W_{20}(\theta)=\left(W_{20}^{(1)}(\theta), W_{20}^{(2)}(\theta), W_{20}^{(3)}(\theta)\right)^{T}$ and $W_{11}(\theta)=\left(W_{11}^{(1)}(\theta), W_{11}^{(2)}(\theta), W_{11}^{(3)}(\theta)\right)^{T}$. By computing, we obtained

$W_{20}(\theta)=-\frac{g_{20}}{i \omega_{0} \tau_{0}} q(\theta)-\frac{\bar{g}_{02}}{3 i \omega_{0} \tau_{0}} \bar{q}(\theta)+E_{1} \mathrm{e}^{2 i \omega_{0} \tau_{0} \theta}$,

and 
$W_{11}(\theta)=\frac{g_{11}}{i \omega_{0} \tau_{0}} q(0) \mathrm{e}^{i \omega_{0} \tau_{0} \theta}-\frac{\bar{g}_{11}}{i \omega_{0} \tau_{0}} \bar{q}(0) \mathrm{e}^{-i \omega_{0} \tau_{0} \theta}+E_{2}$

where $E_{1}=\left(E_{1}^{(1)}, E_{1}^{(2)}, E_{1}^{(3)}\right)$ and $E_{2}=\left(E_{2}^{(1)}, E_{2}^{(2)}, E_{2}^{(3)}\right) \in \mathfrak{R}^{3}$ are the constant vectors, which can be determined by using [51].

Therefore,

$$
E_{1}=\frac{2}{M_{1}}\left(\begin{array}{c}
-\frac{r}{K}-\frac{\delta_{1} b_{1} c_{1} x^{*} y^{*}}{\gamma_{1}^{3}}+\frac{\delta_{2} c_{1} \alpha_{2}}{\gamma_{1}^{2}} \\
\frac{\delta_{1} e_{1} b_{1} c_{1} x^{*} y^{*} \mathrm{e}^{-2 i \omega_{0} \tau_{0}}}{\gamma_{1}^{3}}-\frac{\delta_{2} e_{1} c_{1} \alpha_{2} \mathrm{e}^{-2 i \omega_{0} \tau_{0}}}{\gamma_{1}^{2}} \\
\left.+\frac{\delta_{4} c_{2} \alpha_{2} \alpha_{3}}{\gamma_{2}^{2}}-\frac{\delta_{3} b_{2} c_{2} y^{*} z^{*} \alpha_{2}^{2}}{\gamma_{2}^{3}}\right\} \\
\frac{\delta_{3} e_{2} b_{2} c_{2} y^{*} z^{*} \alpha_{2}^{2}}{\gamma_{2}^{3}}-\frac{\delta_{4} e_{2} c_{2} \alpha_{2} \alpha_{3}}{\gamma_{2}^{2}}
\end{array}\right\} .
$$

Solving this system for $E_{1}$, we have

$E_{1}^{(1)}$

$$
=\frac{2}{M_{1}}\left|\begin{array}{ccc}
-\frac{r}{K}-\frac{\delta_{1} b_{1} c_{1} x^{*} y^{*}}{\gamma_{1}^{3}}+\frac{\delta_{2} c_{1} \alpha_{2}}{\gamma_{1}^{2}} & \frac{c_{1} x^{*}}{\gamma_{1}} & 0 \\
\delta_{1} e_{1} b_{1} c_{1} x^{*} y^{*} \mathrm{e}^{-2 i \omega_{0} \tau_{0}} & -\frac{\delta_{2} e_{1} c_{1} \alpha_{2} \mathrm{e}^{-2 i \omega_{0} \tau_{0}}}{\gamma_{1}^{3}} & 2 i \omega_{0}-\frac{\delta_{4} c_{2} z^{*}}{\gamma_{2}^{2}} \\
+\frac{\delta_{4} c_{2} \alpha_{2} \alpha_{3}}{\gamma_{2}^{2}}-\frac{\delta_{3} b_{2} c_{2} y^{*} z^{*} \alpha_{2}^{2}}{\gamma_{2}^{3}} & +d_{1}-\frac{\mathrm{e}^{2 i \omega_{0} \tau_{0} e_{1} c_{1} x^{*}}}{\gamma_{1}} & \gamma_{2} \\
\frac{\delta_{3} e_{2} b_{2} c_{2} y^{*} z^{*} \alpha_{2}^{2}}{\gamma_{2}^{3}}-\frac{\delta_{4} e_{2} c_{2} \alpha_{2} \alpha_{3}}{\gamma_{2}^{2}} & \frac{\delta_{4} e_{2} c_{2} z^{*}}{\gamma_{2}^{2}} & 2 i \omega_{0}
\end{array}\right|,
$$

$E_{1}^{(2)}$

$$
=\frac{2}{M_{1}}\left|\begin{array}{ccc}
2 i \omega_{0}+\frac{r x^{*}}{K}-\frac{2 b_{1} c_{1} x^{* 2} y^{*}}{\gamma_{1}^{2}} & -\frac{r}{K}-\frac{\delta_{1} b_{1} c_{1} x^{*} y^{*}}{\gamma_{1}^{3}}+\frac{\delta_{2} c_{1} \alpha_{2}}{\gamma_{1}^{2}} & 0 \\
\frac{\mathrm{e}^{2 i \omega_{0} \tau_{0}} \delta_{2} e_{1} c_{1} y^{*}}{\gamma_{1}^{2}} & \frac{\delta_{1} e_{1} b_{1} c_{1} x^{*} y^{*} \mathrm{e}^{-2 i \omega_{0} \tau_{0}}}{\gamma_{1}^{3}}-\frac{\delta_{2} e_{1} c_{1} \alpha_{2} \mathrm{e}^{-2 i \omega_{0} \tau_{0}}}{\gamma_{1}^{2}} & c_{c_{2} y^{*}} \\
\gamma_{2} c_{2} \alpha_{2} \alpha_{3} & \frac{\delta_{3} b_{2} c_{2} y^{*} z^{*} \alpha_{2}^{2}}{\gamma_{2}^{2}}-\frac{\delta_{2}^{3}}{\gamma_{3}} \\
0 & \frac{\delta_{3} e_{2} b_{2} c_{2} y^{*} z^{*} \alpha_{2}^{2}}{\gamma_{2}^{3}}-\frac{\delta_{4} e_{2} c_{2} \alpha_{2} \alpha_{3}}{\gamma_{2}^{2}} & 2 i \omega_{0}
\end{array}\right|,
$$

$E_{1}^{(3)}$

$$
=\frac{2}{M_{1}}\left|\begin{array}{ccc}
2 i \omega_{0}+\frac{r x^{*}}{K}-\frac{2 b_{1} c_{1} x^{* 2} y^{*}}{\gamma_{1}^{2}} & \frac{c_{1} x^{*}}{\gamma_{1}} & -\frac{r}{K}-\frac{\delta_{1} b_{1} c_{1} x^{*} y^{*}}{\gamma_{1}^{3}}+\frac{\delta_{2} c_{1} \alpha_{2}}{\gamma_{1}^{2}} \\
\frac{\mathrm{e}^{2 i \omega_{0} \tau_{0}} \delta_{2} e_{1} c_{1} y^{*}}{\gamma_{1}^{2}} & 2 i \omega_{0}-\frac{\delta_{4} c_{2} z^{*}}{\gamma_{2}^{2}} & \frac{\delta_{1} e_{1} b_{1} c_{1} x^{*} y^{*} \mathrm{e}^{-2 i \omega_{0} \tau_{0}}}{\gamma_{1}^{3}}-\frac{\delta_{2} e_{1} c_{1} \alpha_{2} \mathrm{e}^{-2 i \omega_{0} \tau_{0}}}{\gamma_{1}^{2}} \\
0 & +d_{1}-\frac{\mathrm{e}^{2 i \omega_{0} \tau_{0} e_{1} c_{1} x^{*}}}{\gamma_{1}} & +\frac{\delta_{4} c_{2} \alpha_{2} \alpha_{3}}{\gamma_{2}^{2}}-\frac{\delta_{3} b_{2} c_{2} y^{*} z^{*} \alpha_{2}^{2}}{\gamma_{2}^{3}} \\
\frac{\delta_{4} e_{2} c_{2} z^{*}}{\gamma_{2}^{2}} & \frac{\delta_{3} e_{2} b_{2} c_{2} y^{*} z^{*} \alpha_{2}^{2}}{\gamma_{2}^{3}}-\frac{\delta_{4} e_{2} c_{2} \alpha_{2} \alpha_{3}}{\gamma_{2}^{2}}
\end{array}\right|,
$$


where

$M_{1}$

$$
=\left|\begin{array}{ccc}
2 i \omega_{0}+\frac{r x^{*}}{K}-\frac{2 b_{1} c_{1} x^{* 2} y^{*}}{\gamma_{1}^{2}} & \frac{c_{1} x^{*}}{\gamma_{1}} & 0 \\
\frac{\mathrm{e}^{2 i \omega_{0} \tau_{0}} \delta_{2} e_{1} c_{1} y^{*}}{\gamma_{1}^{2}} & 2 i \omega_{0}-\frac{\delta_{4} c_{2} z^{*}}{\gamma_{2}^{2}}+d_{1}-\frac{\mathrm{e}^{2 i \omega_{0} \tau_{0}} e_{1} c_{1} x^{*}}{\gamma_{1}} & \frac{c_{2} y^{*}}{\gamma_{2}} \\
0 & \frac{\delta_{4} e_{2} c_{2} z^{*}}{\gamma_{2}^{2}} & 2 i \omega_{0}
\end{array}\right| .
$$

Similarly,

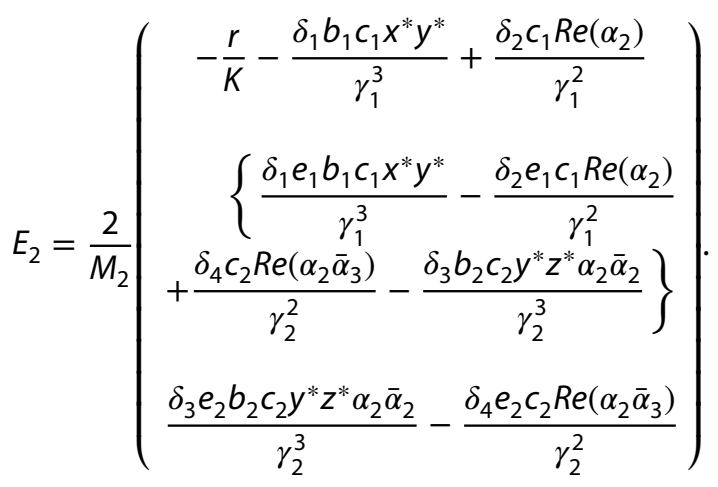

Solving this system for $E_{2}$, we have

$E_{2}^{(1)}$

$$
=\frac{2}{M_{2}}\left|\begin{array}{ccc}
-\frac{r}{K}-\frac{\delta_{1} b_{1} c_{1} x^{*} y^{*}}{\gamma_{1}^{3}}+\frac{\delta_{2} c_{1} \operatorname{Re}\left(\alpha_{2}\right)}{\gamma_{1}^{2}} & \frac{c_{1} x^{*}}{\gamma_{1}} & 0 \\
\frac{\delta_{1} e_{1} b_{1} c_{1} x^{*} y^{*}}{\gamma_{1}^{3}}-\frac{\delta_{2} e_{1} c_{1} \operatorname{Re}\left(\alpha_{2}\right)}{\gamma_{1}^{2}} & -\frac{\delta_{4} c_{2} z^{*}}{\gamma_{2}^{2}}+d_{1}-\frac{e_{1} c_{1} x^{*}}{\gamma_{1}} & \frac{c_{2} y^{*}}{\gamma_{2}} c_{2}\left(\alpha_{2} \bar{\alpha}_{3}\right) \\
\frac{\gamma_{2}^{2}}{\gamma_{3}}-\frac{\delta_{3} b_{2} c_{2} y^{*} z^{*} \alpha_{2} \bar{\alpha}_{2}}{\gamma_{2}^{3}} & \frac{\delta_{4} e_{2} c_{2} z^{*}}{\gamma_{2}^{2}} & 0
\end{array}\right|,
$$

$E_{2}^{(2)}$

$$
\begin{aligned}
& \frac{r x^{*}}{K}-\frac{2 b_{1} c_{1} x^{* 2} y^{*}}{\gamma_{1}^{2}}-\frac{r}{K}-\frac{\delta_{1} b_{1} c_{1} x^{*} y^{*}}{\gamma_{1}^{3}}+\frac{\delta_{2} c_{1} \operatorname{Re}\left(\alpha_{2}\right)}{\gamma_{1}^{2}} \quad 0 \\
& =\frac{2}{M_{2}} \mid \begin{array}{c}
\frac{\delta_{2} e_{1} c_{1} y^{*}}{\gamma_{1}^{2}} \\
+\frac{\delta_{4} c_{2} \operatorname{Re}\left(\alpha_{1} c_{1} \bar{\alpha}_{3}\right)}{\gamma_{2}^{3}}-\frac{\delta_{3} b_{2} c_{2} y^{*} z^{*} \alpha_{2} \bar{\alpha}_{2}}{\gamma_{2}^{3}}
\end{array} \\
& 0 \quad \frac{\delta_{3} e_{2} b_{2} c_{2} y^{*} z^{*} \alpha_{2} \bar{\alpha}_{2}}{\gamma_{2}^{3}}-\frac{\delta_{4} e_{2} c_{2} \operatorname{Re}\left(\alpha_{2} \bar{\alpha}_{3}\right)}{\gamma_{2}^{2}} \quad 0
\end{aligned}
$$


and

$E_{2}^{(3)}$

$$
=\frac{2}{M_{2}}\left|\begin{array}{ccc}
\frac{r x^{*}}{K}-\frac{2 b_{1} c_{1} x^{* 2} y^{*}}{\gamma_{1}^{2}} & \frac{c_{1} x^{*}}{\gamma_{1}} & -\frac{r}{K}-\frac{\delta_{1} b_{1} c_{1} x^{*} y^{*}}{\gamma_{1}^{3}}+\frac{\delta_{2} c_{1} \operatorname{Re}\left(\alpha_{2}\right)}{\gamma_{1}^{2}} \\
\frac{\delta_{2} e_{1} c_{1} y^{*}}{\gamma_{1}^{2}} & -\frac{\delta_{4} c_{2} z^{*}}{\gamma_{2}^{2}}+d_{1}-\frac{e_{1} c_{1} x^{*}}{\gamma_{1}} & \frac{\delta_{1} e_{1} b_{1} c_{1} x^{*} y^{*}}{\gamma_{1}^{3}}-\frac{\delta_{2} e_{1} c_{1} \operatorname{Re}\left(\alpha_{2}\right)}{\gamma_{1}^{2}} \\
0 & \frac{\delta_{4} c_{2} \operatorname{Re}\left(\alpha_{2} \bar{\alpha}_{3}\right)}{\gamma_{2}^{2}}-\frac{\delta_{3} b_{2} c_{2} y^{*} z^{*} \alpha_{2} \bar{\alpha}_{2}}{\gamma_{2}^{3}} \\
\frac{\delta_{4} e_{2} c_{2} z^{*}}{\gamma_{2}^{2}} & \frac{\delta_{3} e_{2} b_{2} c_{2} y^{*} z^{*} \alpha_{2} \bar{\alpha}_{2}}{\gamma_{2}^{3}}-\frac{\delta_{4} e_{2} c_{2} \operatorname{Re}\left(\alpha_{2} \bar{\alpha}_{3}\right)}{\gamma_{2}^{2}}
\end{array}\right|,
$$

where

$$
M_{2}=\left|\begin{array}{ccc}
\frac{r x^{*}}{K}-\frac{2 b_{1} c_{1} x^{* 2} y^{*}}{\gamma_{1}^{2}} & \frac{c_{1} x^{*}}{\gamma_{1}} & 0 \\
\frac{\delta_{2} e_{1} c_{1} y^{*}}{\gamma_{1}^{2}} & -\frac{\delta_{4} c_{2} z^{*}}{\gamma_{2}^{2}}+d_{1}-\frac{e_{1} c_{1} x^{*}}{\gamma_{1}} & \frac{c_{2} y^{*}}{\gamma_{2}} \\
0 & \frac{\delta_{4} e_{2} c_{2} z^{*}}{\gamma_{2}^{2}} & 0
\end{array}\right| .
$$

Consequently, we determine the value of $W_{20}(\theta)$ and $W_{11}(\theta)$ from Eqs. (3.49) and (3.50). The value of $g_{21}$ can be expressed by delay and parameters in Eq. (3.48). Hence, we will compute the different values:

$$
\begin{aligned}
c_{1}(0) & =\frac{i}{2 \omega_{0} \tau_{0}}\left(g_{20} g_{11}-2\left|g_{11}\right|^{2}-\frac{\left|g_{02}\right|^{2}}{3}\right)+\frac{g_{21}}{2}, \\
\mu_{2} & =-\frac{\mathfrak{R}\left\{c_{1}(0)\right\}}{\mathfrak{R}\left\{\lambda^{\prime}\left(\tau_{0}\right)\right\}}, \\
\beta_{2} & =2 \mathfrak{R}\left\{c_{1}(0)\right\}, \\
T_{2} & =-\frac{\mathfrak{\Im}\left\{c_{1}(0)\right\}+\mu_{2} \mathfrak{\Im}\left\{\lambda^{\prime}\left(\tau_{0}\right)\right\}}{\omega_{0} \tau_{0}} .
\end{aligned}
$$

The above values determine the bifurcating periodic solution at the critical value $\tau_{0}$ in the center manifold.

Theorem 4 For Eq. (3.51), we have following results:

(i) Direction of the Hopf bifurcation is supercritical (subcritical) if $\mu_{2}>0\left(\mu_{2}<0\right)$.

(ii) Stability of the bifurcating periodic solution is stable (unstable) if $\beta_{2}<0\left(\beta_{2}>0\right)$.

(iii) Period of the bifurcating periodic solution increases (decreases) if $T_{2}>0\left(T_{2}<0\right)$.

\section{Results}

In this section, we perform numerical simulations to understand the mechanism that avoid the extinction of species and stabilizes the chaotic system. For that purpose, we have plotted the bifurcation diagram, time series and phase portrait of the model system (2.1). We choose the following set of parameters value;

$$
\begin{aligned}
r & =0.7, \\
K & =28 \\
c_{1} & =0.65 \\
c_{2} & =0.45 \\
b_{1} & =0.025 \\
b_{2} & =0.035 \\
m_{1} & =15 \\
m_{2} & =13 \\
d_{1} & =0.23 \\
d_{2} & =0.15 \\
e_{1} & =0.9 \\
e_{2} & =0.99 .
\end{aligned}
$$

Table 1 Dynamical outcome of model system (2.1) for different parameters range at $\tau=0$

\begin{tabular}{ll}
\hline Range of parameters & System behavior \\
\hline $0.25 \leq r \leq 0.41$ & Stable \\
$0.41<r \leq 0.75$ & Limit cycle \\
$32.5 \leq K \leq 36$ & Chaos \\
$0.035 \leq b_{1} \leq 0.06$ & Chaos \\
$0.057 \leq b_{2} \leq 0.07$ & Chaos \\
\hline
\end{tabular}




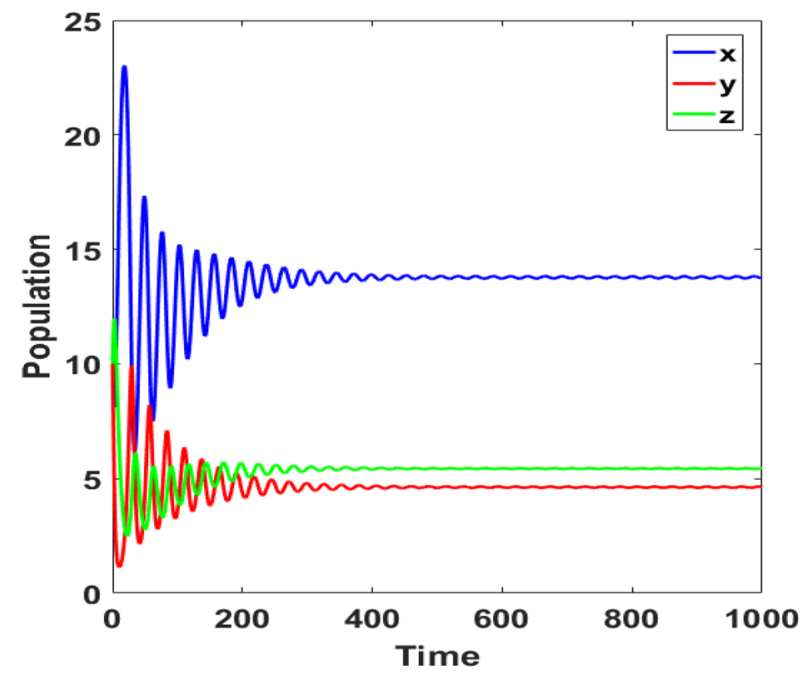

(a)

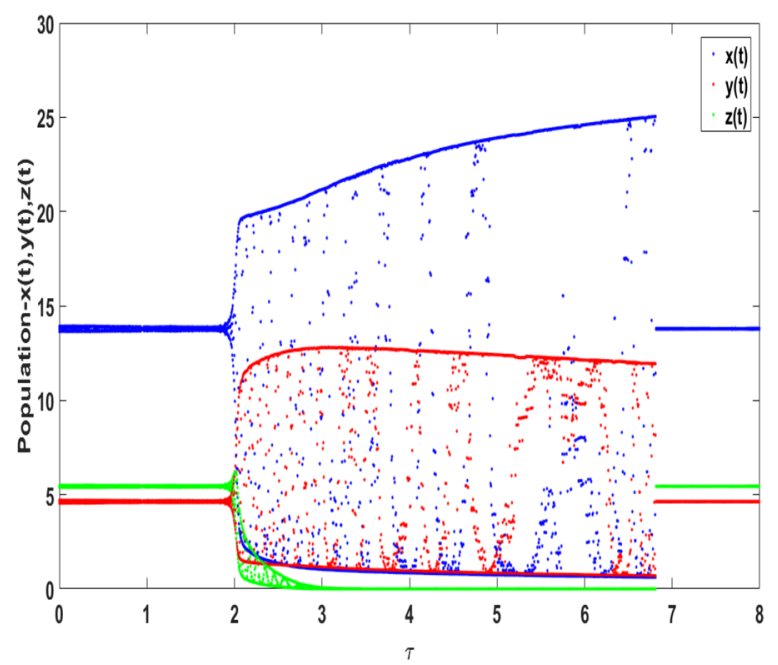

(b)

Fig. 1 a Time series representation for model system (2.1) with $r=0.3$ at $\tau=0$, b bifurcation diagram of time lag $\tau$ versus population density $x(t), y(t)$, and $z(t)$ for model system (2.1) with $r=0.3$

For the above set of parameters value, we get the value of the interior equilibrium point $E^{*}\left(x^{*}, y^{*}, z^{*}\right)=(13.7777,4.6440,5.4312)$ which is locally asymptotically stable for $r=0.3$ (c.f., Fig. 1a). We have substantiated the dynamical behavior of the model system (2.1) without delay for different parameters range which are enlisting in Table 1 and observe the effect of time delay in the same parametric value.

Taking the above set of parameter values with $r=0.3$, we have plotted the bifurcation diagram between time lag $\tau$ and population density $x(t), y(t), z(t)$ in the interval $\tau \in[0,8]$ in which all the population bifurcates for the two values of time delay, i.e., $\tau_{0}^{1}=1.89$ and $\tau_{0}^{2}=6.825$ (c.f., Fig. 1b). To the justification of bifurcation diagram of Fig. $1 \mathrm{~b}$, we have plotted time series in which one can see that model system (2.1) remains stable when $\tau<\tau_{0}^{1}=1.89$ (c.f., Fig. 2a) and as we increase the value of $\tau$ by $\tau_{0}^{1}$, system becomes unstable (c.f., Fig. 2 b) and at the critical value $\tau=\tau_{0}^{1}=1.89$, model system (2.1) undergoes Hopf bifurcation. Further, we can see the extinction in top predator after certain oscillation in the range of $\tau$, i.e., top predator shows limit cycle behavior in the range $1.89 \leq \tau<2.72$ and after that extinction has been observed in the range $2.72 \leq \tau<6.825$ (c.f., Fig. 2c), whereas limit cycle behavior is obtained for prey and middle predator in the range $1.89 \leq \tau<6.825$. When $\tau \geq \tau_{0}^{2}=6.825$, limit cycle behavior disappears through second Hopf bifurcation and system again shows stable behavior for all the population (c.f., Fig. 2d). Now, we choose $r=0.7$ and kept other parameters are fixed. A limit cycle behavior is observed for model system (2.1) with $\tau=0$ (c.f., Fig. 3a). By incorporating $\tau$ at this situation, a stable focus is observed by increasing the value of $\tau$ which is shown by the bifurcation diagram (c.f., Fig. 3b). It is also justified by the phase space diagram that $\tau=1.5$, the system shows limit cycle behavior and $\tau=4$, the system shows a stable focus (c.f., Fig. 4).

To understand the dynamical behavior of model system (2.1) with respect to carrying capacity $K$, we have chosen $K=33$ and kept other parameters are fixed. Irregular oscillation is observed for model system (2.1) with $\tau=0$ (c.f., Fig. 5a). We have chosen a window of $0.0 \leq \tau \leq 7.0$ and plotted the bifurcation diagram between time delay and successive maxima of $x, y, z$ in the range [0.0,35.0], [0.0, 25.0] and [0.0, 25.0], respectively, at $K=33$ (c.f., Fig. 5b-d). To verify the dynamical behavior of Fig. $5 b-d$, we have also drawn time series and phase space diagram for four different conditions, i.e., stable focus, periodic solution of order one, periodic solution of order two and chaos. When $\tau<0.25$, the system shows chaotic behavior. As the value of the time delays $\tau$ increases, the system shows periodic solution of order two, then reduces to a limit cycle and finally shows a stable focus for a large value of $\tau$. Fig. $6 a-d$ shows different nature of system for different values of $\tau$, i.e., $\tau=0.25,1.5,2.8$ and 5 , respectively. Similarly, we have chosen a window of $0 \leq \tau \leq 9$ at $b_{1}=0.05$ and $0 \leq \tau \leq 6$ at $b_{2}=0.06$ and plotted the bifurcation diagram between time delay and successive maxima of $x, y, z$, respectively (c.f., Fig. 7b-d and 8b-d). In Figs. 7 and 8, one can see for the increasing value of time delay $\tau$ stabilizes the chaotic behavior of the model system. This result shows that the obtained outcomes are highly affected in the presence of time delay $\tau$. 


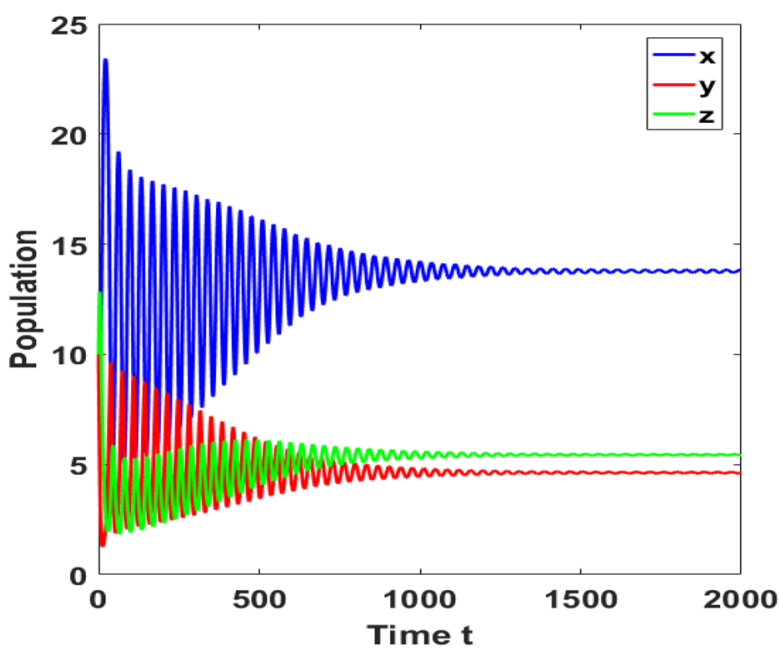

(a)

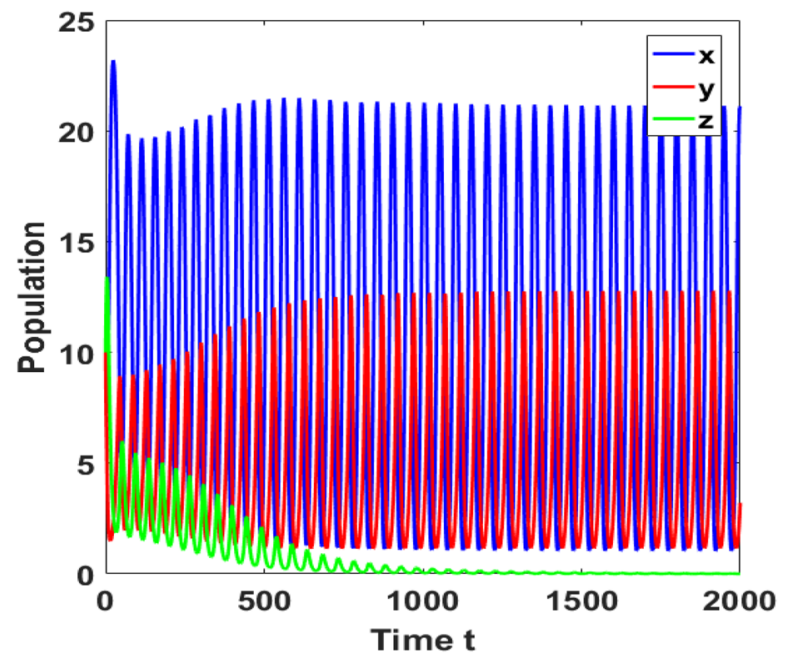

(c)

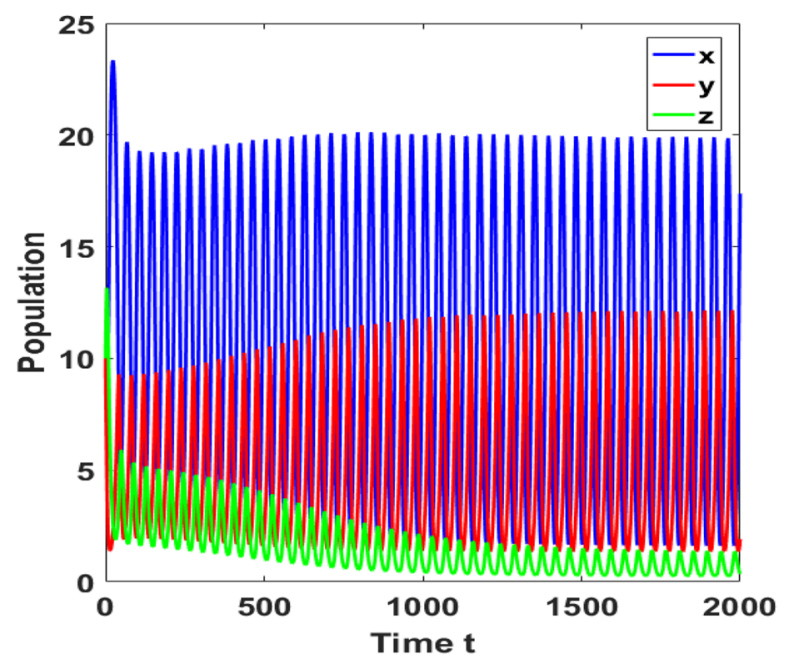

(b)

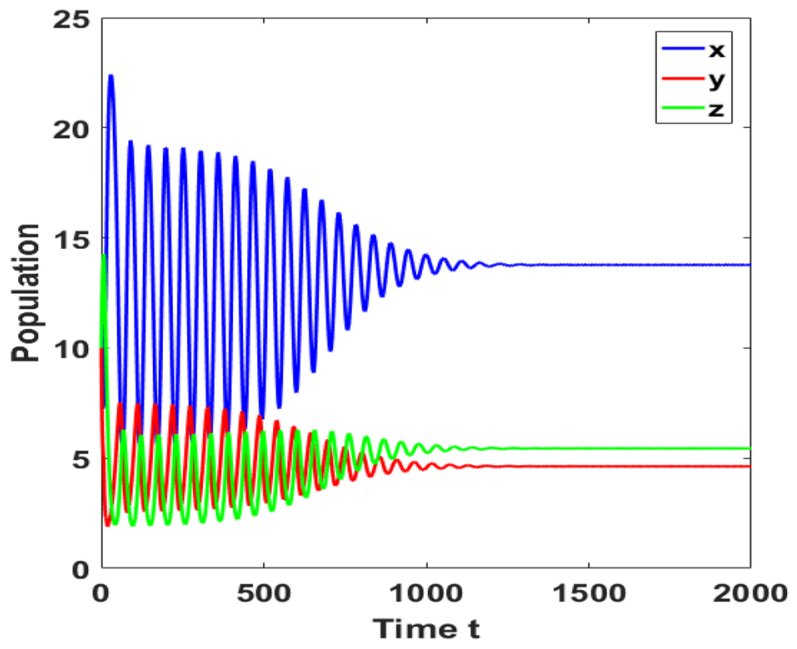

(d)

Fig. 2 Time series for model system (2.1) with $r=0.3$ at $\mathbf{a} \tau=1.5, \mathbf{b} \tau=2.3, \mathbf{c} \tau=3, \mathbf{d} \tau=7$

\section{Discussions}

In this article, we have explored the dynamics through the defense mechanism adopted by phytoplankton and zooplankton in the presence of fish population. The influence of time delay is studied theoretically with numerical investigation. It has been observed that some time delay is required in the DVM strategy adopted by zooplankton. We have summarized and made a needful comparison of the present study with some previous studies.

1. A plankton-fish interaction with the hybridization of Holling type III and $\mathrm{MH}$-type function response is studied by Raw and Mishra [53], but the effect of time delay on the species dynamics has been neglected.
Assume that time delay in the form of gestation, predation, digestion, traveling, etc., fascinated the area of plankton dynamics.

2. Sharma et al. [54] investigated the interaction between toxin-producing phytoplankton (TPP) and zooplankton with the assumption that zooplankton predation must take some time delay $\tau$. Zhang and Rehim [55] investigated a toxic phytoplankton-zooplankton system with the fact that toxin liberation must take some time variation $\tau$ and observed that time delay complicates the stability and bifurcation of the system. But the influence of top predator on the system dynamics has ignored. A delayed prey-predator model in the presence of top predator is more appropriate to 


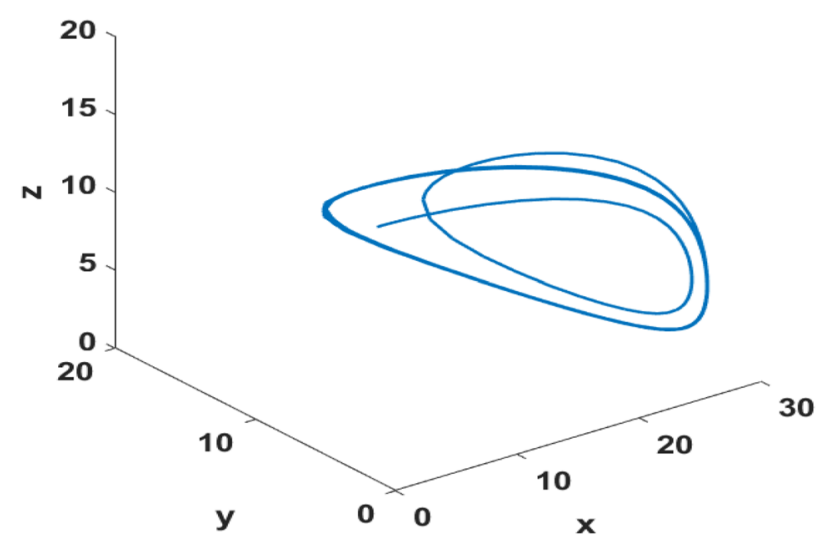

(a)

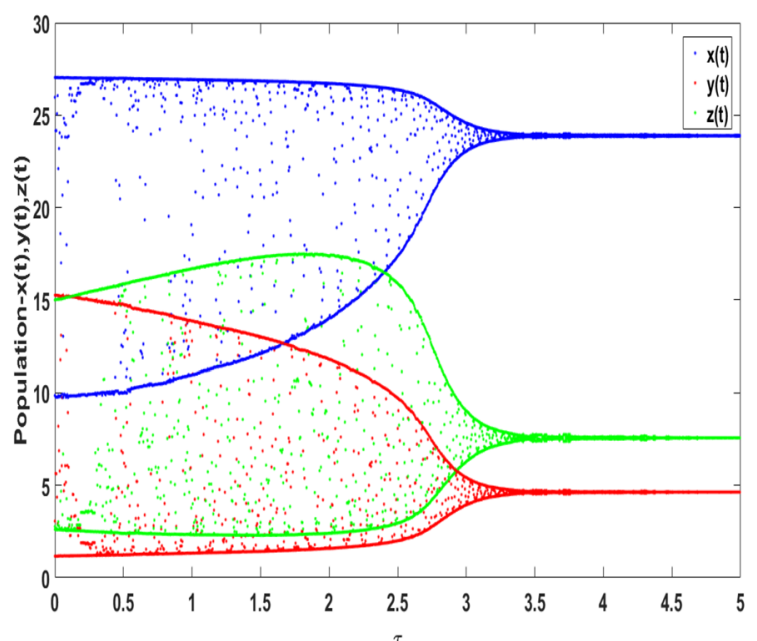

(b)

Fig. 3 a Phase space representation for model system (2.1) with $r=0.7$ at $\tau=0$, $\mathbf{b}$ bifurcation diagram of time lag $\tau$ versus population density $x(t), y(t)$, and $z(t)$ for model system (2.1) with $r=0.7$

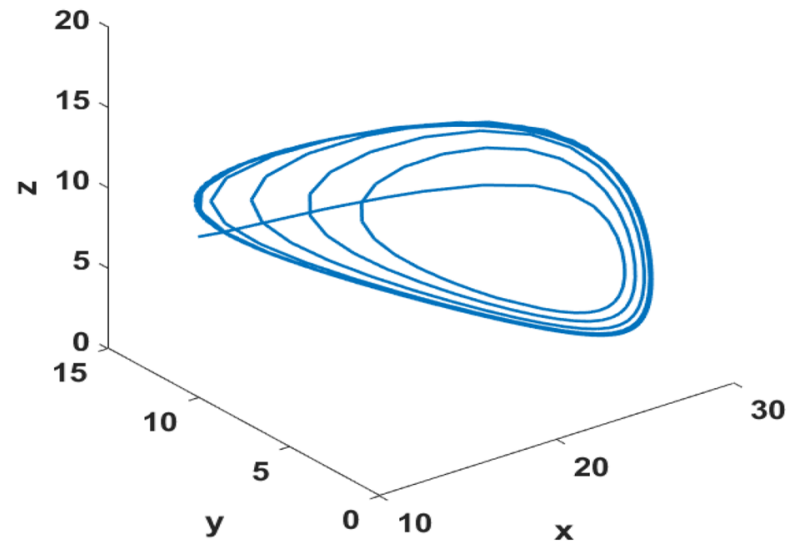

(a)

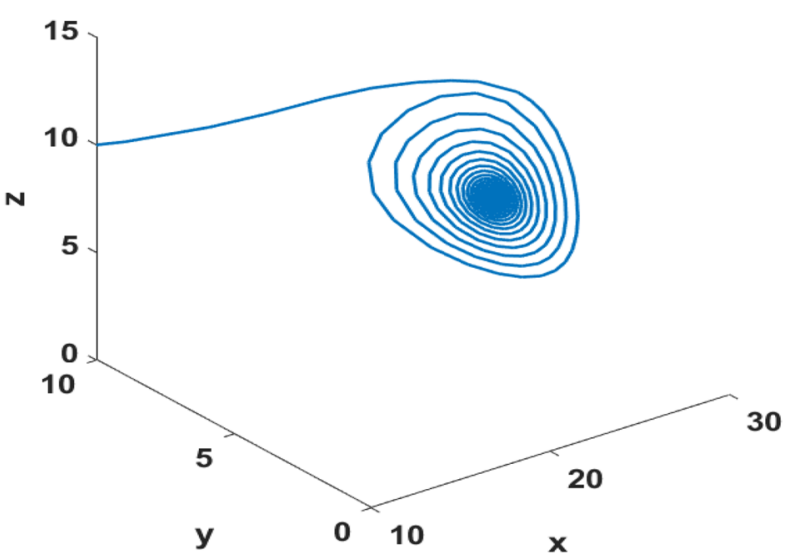

(b)

Fig. 4 a Phase space representation for model system (2.1) with $r=0.7$ at $\mathbf{a} \tau=1.5, \mathbf{b} \tau=4$

explore the consequences of the mechanism adopted by them.

3. Some existing studies have focused on studying the toxin-produced phytoplankton by using an independent parameter (toxication rate) $[7,46]$, but the current work has been adopted a distinct approach for analyzing the relationship among toxin-produced phytoplankton, zooplankton and planktivorous fish population by assuming $\mathrm{MH}$-type functional response.

4. We have extensively studied the dynamics of the plankton-fish interaction model (2.1) with time delay corresponding to the all feasible equilibria. Zooplank- ton are usually tried to skip those area where the prey density is very high due to adverse effect of toxic substance released by phytoplankton. Motivated by these observations, we have modeled the predator feeding through $\mathrm{MH}$-type functional response which ensures depletion in per capita predation rate of zooplankton at sufficiently thick phytoplankton density due to toxicity.

5. Agrawal et al. [56] focused on prey-predator problems considering prey, specialist predator and generalist predator where the generalist predator grows sexually. They used Holling type IV and Beddington-DeAngelis- 


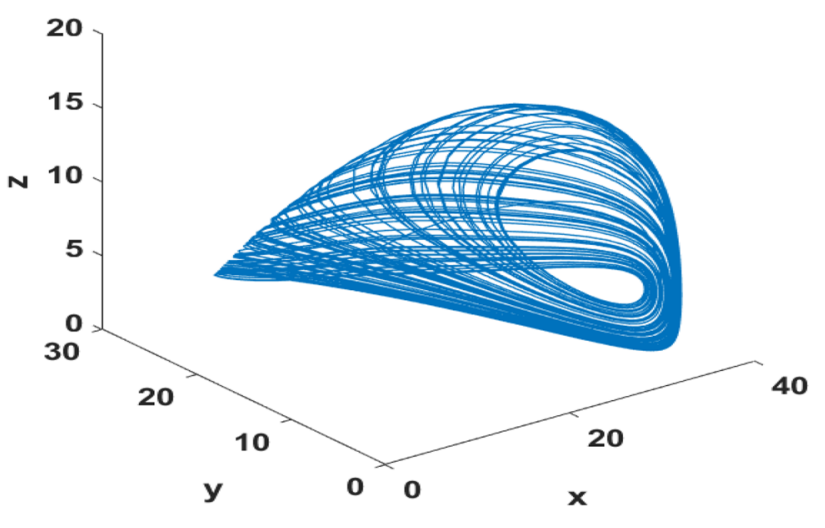

(a)

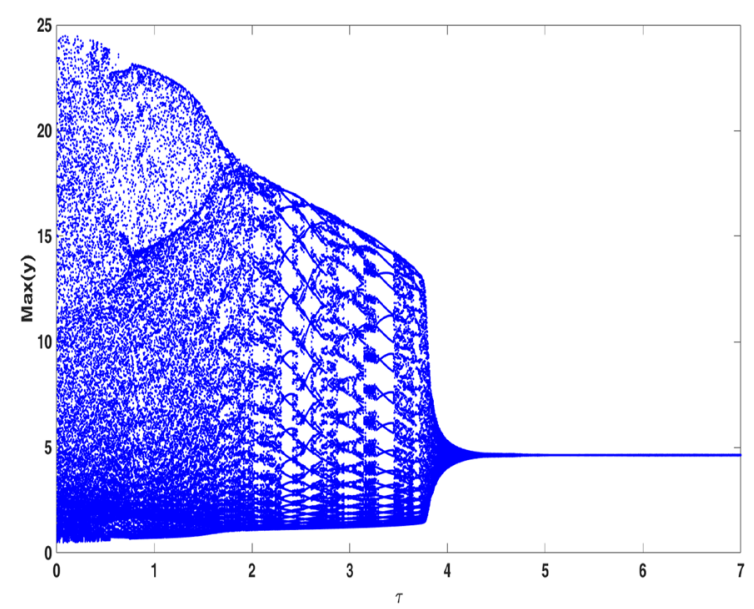

(c)

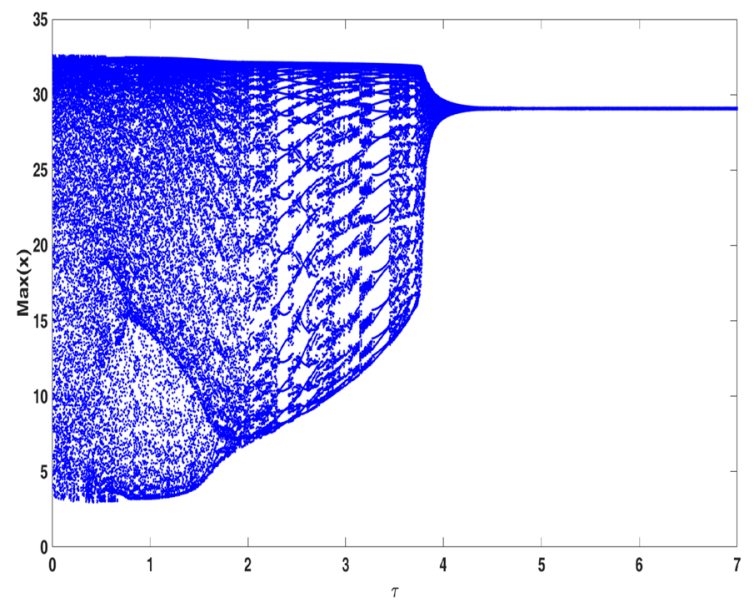

(b)

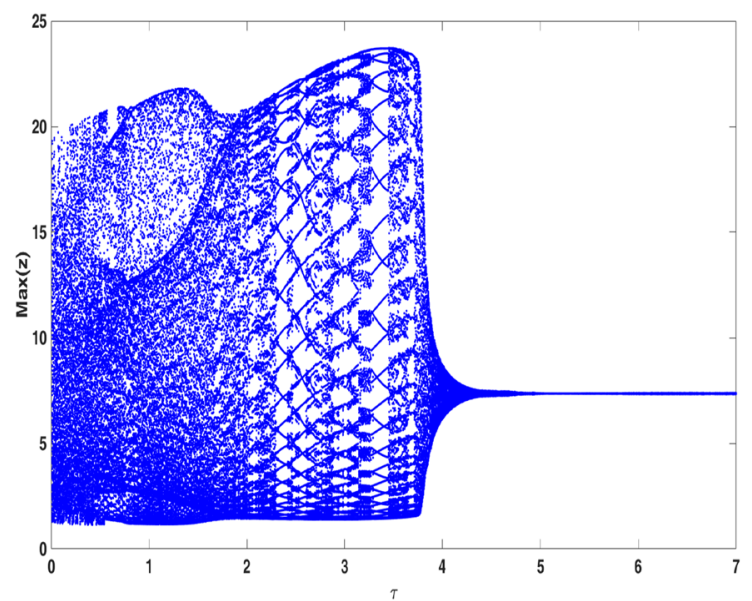

(d)

Fig. 5 a Time series representation for model system (2.1) with $K=33$ at $\tau=0, \mathbf{b}$-d bifurcation diagram of time lag $\tau$ versus population density $x(t), y(t)$, and $z(t)$ for model system (2.1) with $K=33$

type functional response to describe the relationship between prey and predators and observed a scenario of double Hopf bifurcation. But, here we have chosen all the species of specialist type and concentrated on the defense mechanism which strongly helps in survivorship of phytoplankton and zooplankton and becomes a reason for fish extinction.

6. Table 1 demonstrates the dynamical outcome corresponding to the different parameters' restriction in the absence of delay, mainly examining the role of $r$, $K, b_{1}$ and $b_{2}$. Figure $1 \mathrm{~b}$ equivalently reveals the signifi- cance of defense ability with the importance of time delay $\tau$. If the phytoplankton's growth rate is sufficiently small, then one can clearly see the extinction in the fish population with the variation in $\tau$, and if we slightly increase the value of $\tau$ after a certain interval, all the species stabilize together. This result is agreeable with a real ecosystem. Consequently, our model system exhibits rich dynamics which has not been overlooked earlier in such models, which also shows the existence of double Hopf bifurcation. Figure $3 \mathrm{~b}$ shows the small change in phytoplankton's growth 

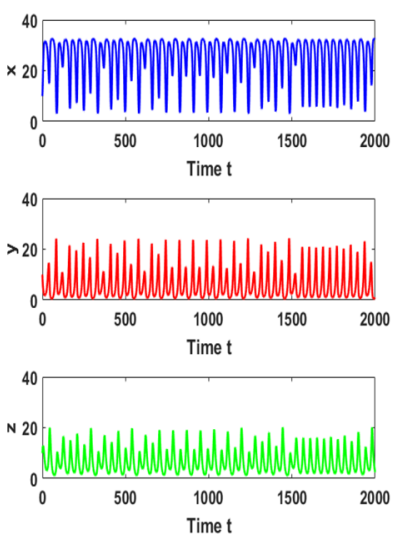

(a)
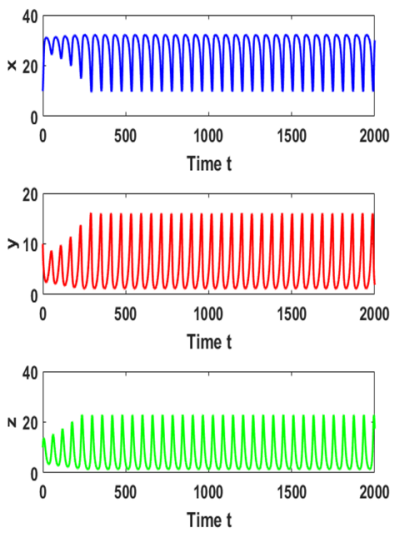

(c)
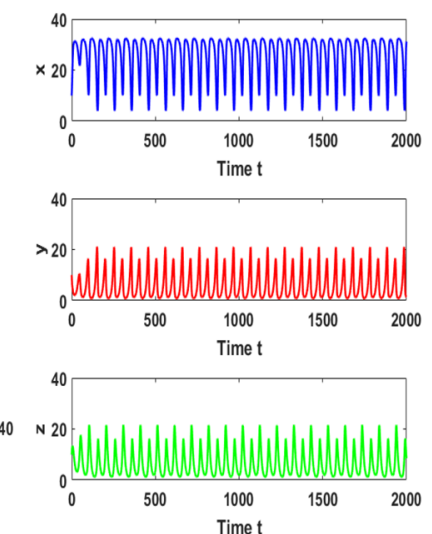

(b)
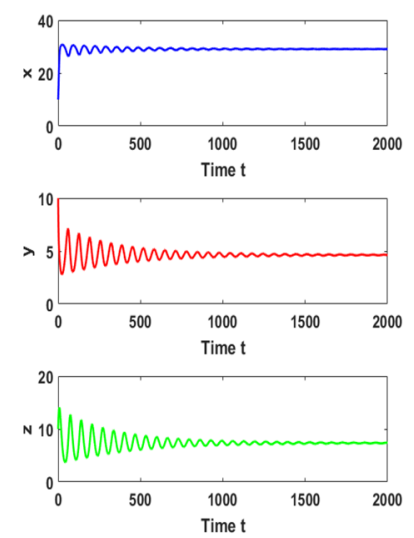

(d)
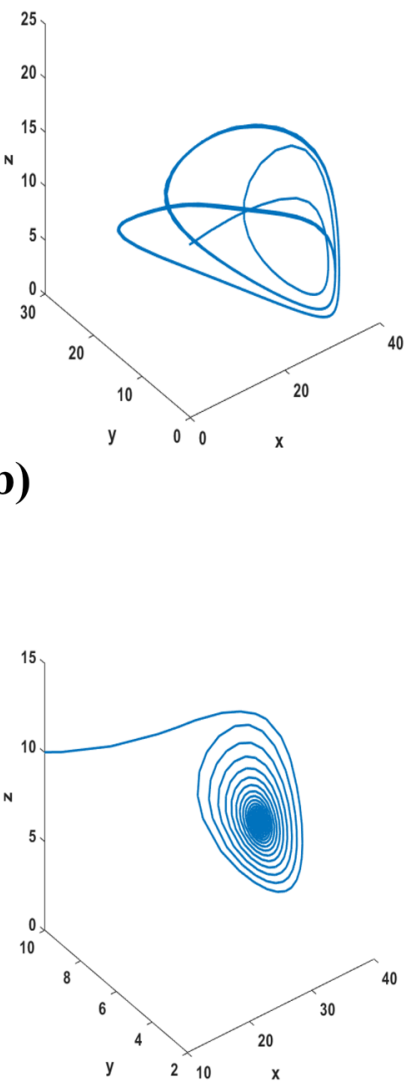

Fig. 6 Time series and phase space for model system (2.1) with $K=33$ at $\mathbf{a} \tau=0.25, \mathbf{b} \tau=1.5, \mathbf{c} \tau=2.8, \mathbf{d} \tau=5$

rate destabilized the dynamics and with variation in $\tau$ stabilizes the whole plankton dynamics.

7. Our numerical investigation also shows under the consequences of time delay, the system exhibits chaotic and periodic solution of order two, periodic solution of order one (i.e., limit cycle) and stable focus behavior with the increasing value of $\tau$ (c.f., Figs. $5 b-d, 7 b-$ $d, 8 b-d)$. In general, time delay enhances the system complexity $[44,57]$, but in our case, increasing the value of time delay reduces the complexity through period-doubling cascade, and finally, the whole system becomes stable. This finding reveals the importance of time delay in a real biological system.

Based on the current study, the dependence of plankton-fish interaction on time delay is significantly more realistic than a system without time delay. We also noticed a vital change in plankton-fish interaction for varying time delay parameter $\tau$ by observing chaos, limit cycle, stable focus, extinction behavior, Hopf bifurcation and double Hopf bifurcation.

\section{Conclusion}

In this paper, we have proposed a tri-tropic food chain model for plankton-fish dynamics with Monod-Haldanetype functional response. The choice of such functional response fits significantly as a repulsive effect on zooplankton due to the toxicity of phytoplankton. Similarly, zooplankton uses diel vertical migration as a defensive strategy. We have studied the effect of time delay and defense mechanism adopted by plankton. The dynamical behavior for the model system is analyzed in the presence of time delay. The local stability conditions of all the feasible equilibria have been discussed. The interior 


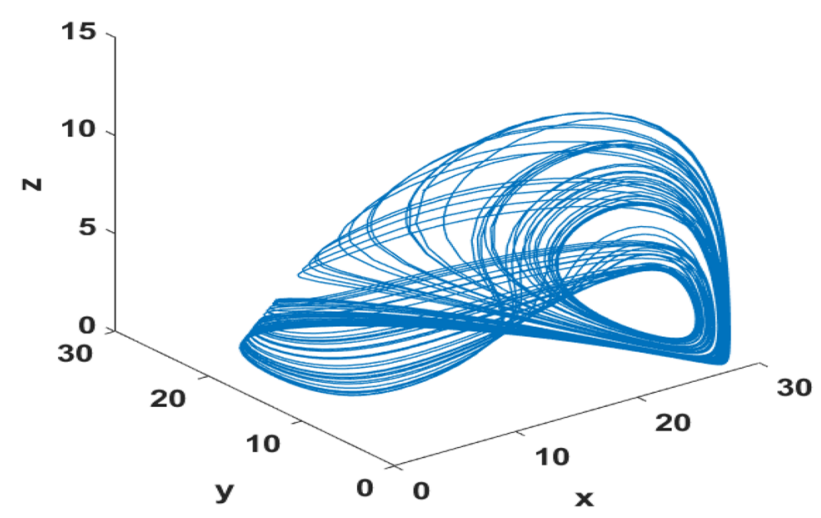

(a)

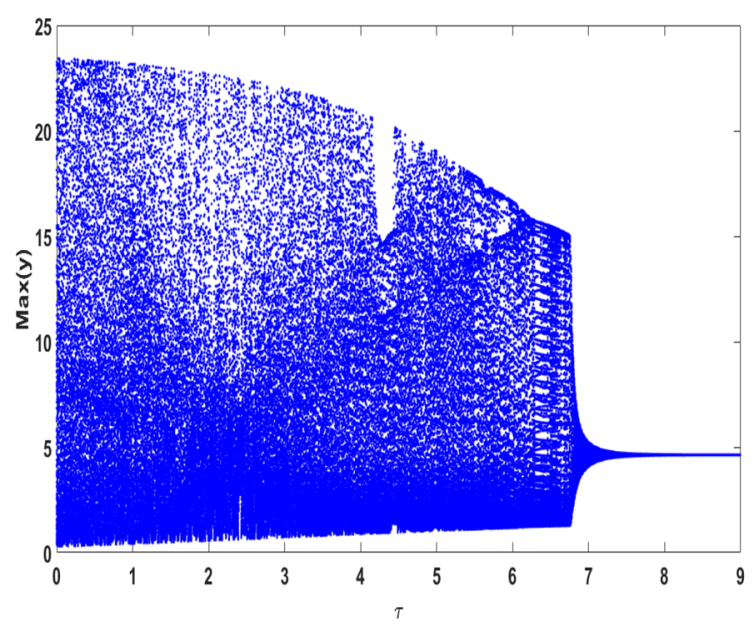

(c)

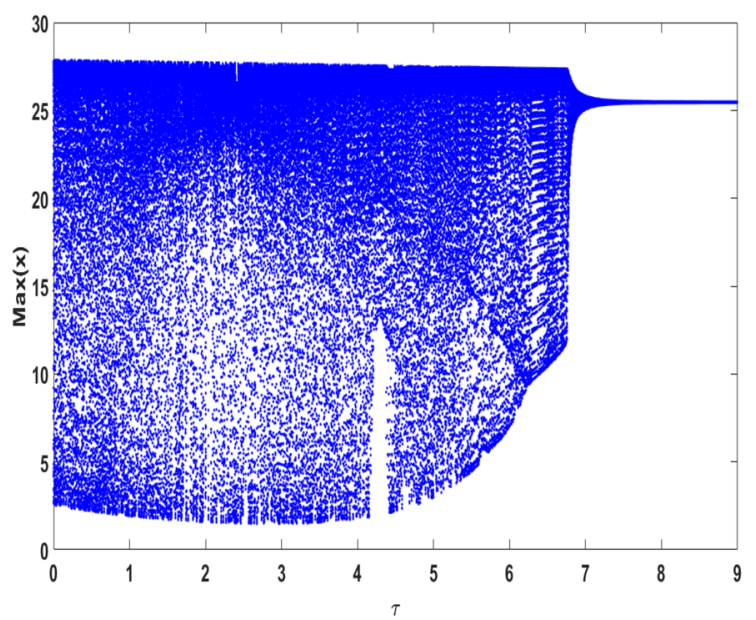

(b)

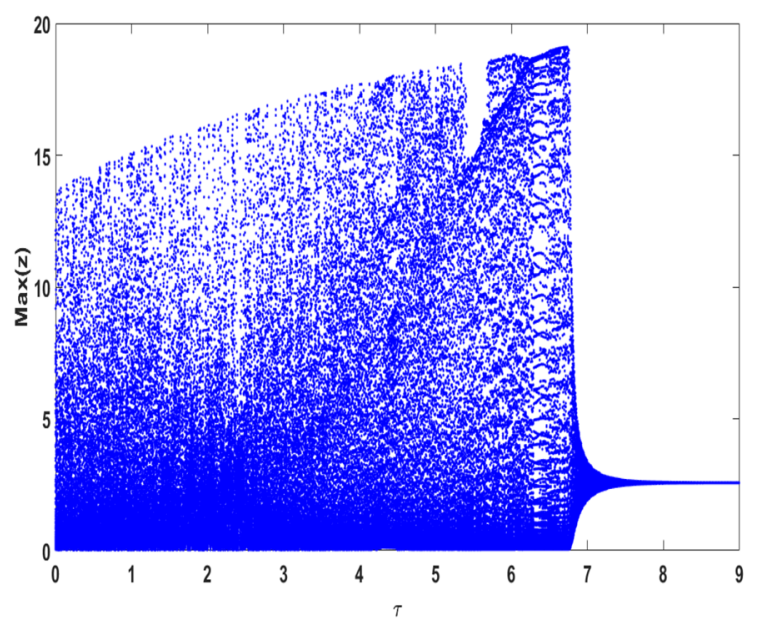

(d)

Fig. 7 a Phase space representation for model system (2.1) with $b_{1}=0.05$ at $\tau=0, \mathbf{b}$ - $\mathbf{d}$ bifurcation diagram of time lag $\tau$ versus population density $x(t), y(t)$, and $z(t)$ for model system (2.1) with $b_{1}=0.05$

equilibrium point $E^{*}\left(x^{*}, y^{*}, z^{*}\right)$ is locally as well as globally asymptotic stable under certain conditions. We have also derived the conditions for stability and direction of the Hopf bifurcation. Our study shows that the defense mechanism adopted by phytoplankton and zooplankton in the presence of time delay plays an important role in the dynamical change of the planktonic system. The ability of defense maintains the survival of prey and middle predator in the presence of the top predator. We observed extinction in top predator due to time delay with moderate value of intrinsic growth rate of prey (c.f., Fig. 1b).
Such extinction can be avoided with increasing the value of intrinsic growth rate (c.f., Fig. 3b). Thus, the intrinsic growth rate of prey (i.e., $r$ ) and time delay (i.e., $\tau$ ) are two important parameters that control the system dynamics. Further, the large value of $\tau$ can stabilize the system as well as help to avoid the extinction of top predators due to the negative effect of defense (c.f., Fig. 2). Some other parameters also influence the system dynamics, and for a different parametric range, chaotic dynamics are observed in the absence of time delay (c.f., Figs. 5a, 7a, 8a). In the absence 


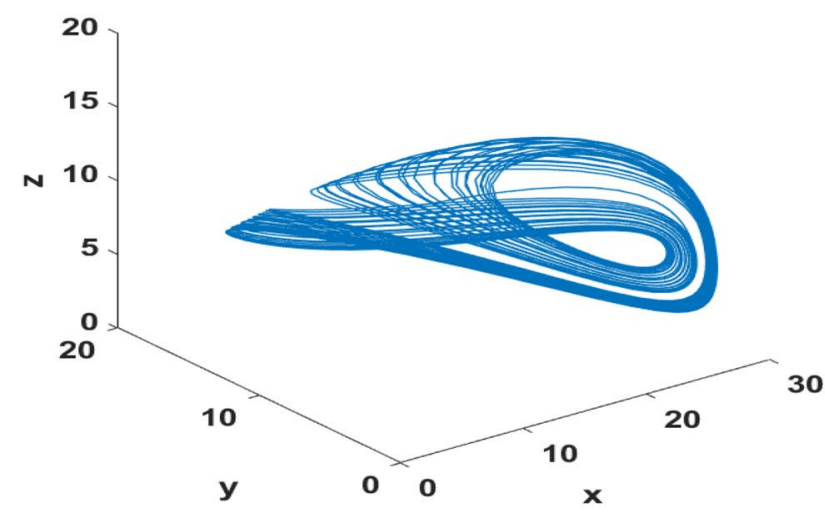

(a)

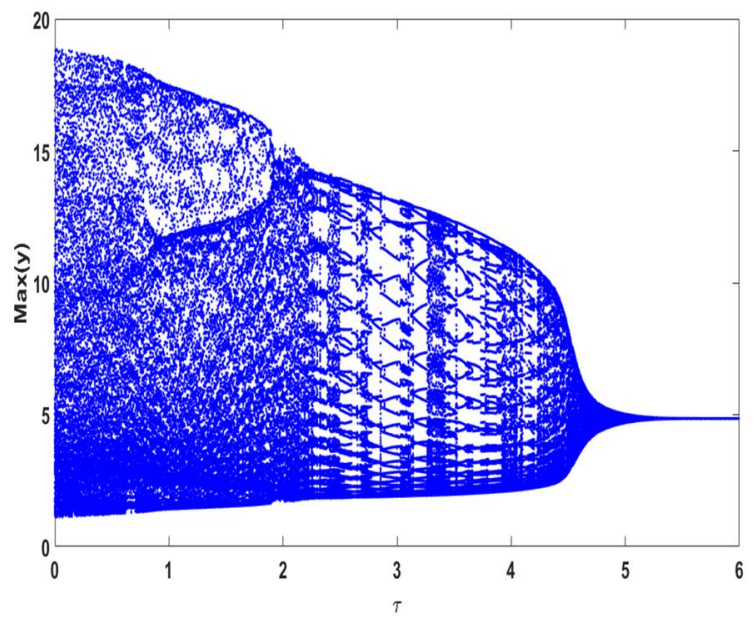

(c)

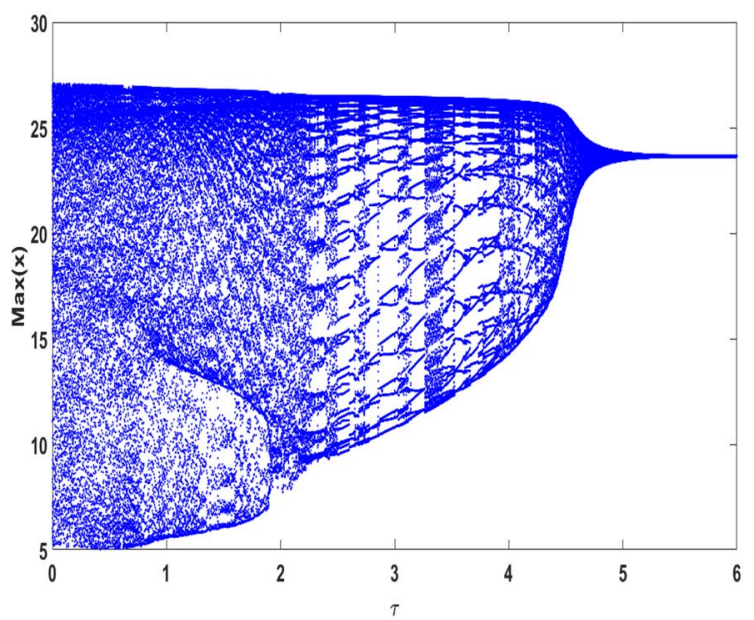

(b)

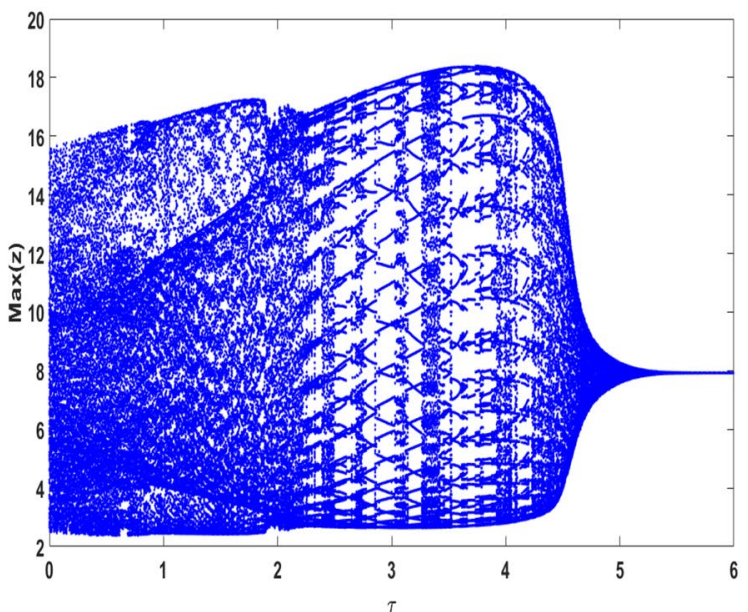

(d)

Fig. 8 a Phase space representation for model system (2.1) with $b_{2}=0.06$ at $\tau=0$, $\mathbf{b}$-d bifurcation diagram of time lag $\tau$ versus population density $x(t), y(t)$, and $z(t)$ for model system (2.1) with $b_{2}=0.06$

of time delay or for smaller values, system shows the chaotic behavior but by increasing the value of $\tau$, the system becomes stable (c.f., Figs. 5b-d, 7b-d, 8b-d). The system shows the positive impact of time delay that stabilizes the chaotic dynamics of the proposed system. If the time delay is varied by some critical value, the interior equilibrium of the system switches from stable to unstable and vice versa. It is remarkable that the occurrence of double Hopf bifurcation depends on the value of $r$ because as we take the value of $r$ as 0.7 or larger, no double Hopf bifurcation scenario is observed. Some features require further investigation as collaborative behavior of time delay with diffusion in the presence of defense mechanism.

Acknowledgements This research work is supported by Science and Engineering Research Board (SERB), Govt. of India, under the Grant No. EMR/2017/000607 to the corresponding author (Nilesh Kumar Thakur). 


\section{Compliance with ethical standards}

Conflict of interest The authors declare that they have no conflict of interest.

\section{References}

1. Tollrian R (1995) Predator-induced morphological defenses: costs, life history shifts, and maternal effects in Daphnia pulex. Ecology 76(6):1691-1705

2. Khare S, Misra OP, Singh C, Dhar J (2011) Role of delay on planktonic ecosystem in the presence of a toxic producing phytoplankton. Int J Differ Equ 2011:1-16

3. Pan Y, Zhang YY, Sun SC (2014) Phytoplankton-zooplankton dynamics vary with nutrients: a microcosm study with the cyanobacterium Coleofasciculus chthonoplastes and cladoceran Moina micrura. J Plankton Res 36(5):1323-1332

4. Donk EV, lanora A, Vos M (2011) Induced defences in marine and freshwater phytoplankton: a review. Hydrobiologia 668(1):3-19

5. Pal S, Chatterjee S, Chattopadhyay J (2007) Role of toxin and nutrient for the occurrence and termination of plankton bloomresults drawn from field observations and a mathematical model. Biosystem 90(1):87-100

6. Chakarborty S, Roy S, Chattopadhyay J (2008) Nutrient-limiting toxin producing and the dynamics of two phytoplankton in culture media: a mathematical model. Ecol Model 213(2):191-201

7. Chakraborty K, Das K (2015) Modeling and analysis of a twozooplankton one-phytoplankton system in the presence of toxicity. Appl Math Model 39(3-4):1241-1265

8. Upadhyay RK, Chattopadhyay J (2005) Chaos to order: role of toxin producing phytoplankton in aquatic systems. Nonlinear Anal: Modell Control 10(4):383-396

9. Lampert W (1993) Ultimate causes of diel vertical migration of zooplankton: new evidence for the predator-avoidance hypothesis. Arch Hydrobiol Beih Ergebn Limnol 39:79-88

10. Cohen JH, Forward RB Jr (2009) Zooplankton diel vertical migration-a review of proximate control. In: Gibson RN, Atkinson RJA, Gordon JDM (eds) Oceanography and marine biology: an annual review, vol 47. CRC Press, Boca Raton, pp 77-110

11. Ohman MD (1990) The demographic benefits of diel vertical migration by zooplankton. Ecol Monogr 60(3):257-281

12. Riessen $H$ (1992) Cost-benefit model for the induction of an antipredator defense. Am Nat 140(2):349-362

13. Sih A (1992) Prey uncertainty and the balance of antipredator and feeding needs. Am Nat 139(5):1052-1069

14. Pietrzak B, Pijanowska J, Dawidowicz P (2017) The efect of temperature and kairomone on Daphnia escape ability: a simple bioassay. Hydrobiologia 798(1):15-23

15. Wang J, Jiang W (2012) Bifurcation and chaos of a delayed predator-prey model with dormancy of predators. Nonlinear Dyn 69(4):1541-1558

16. Malik T, Smith HL (2006) A resource-based model of microbial quiescence. J Math Biol 53(2):231-252

17. Hadeler KP (2007) Quiescent phases and stability. Linear Algebra Appl 428(7):1620-1627

18. Chen X, Wang X (2019) Qualitative analysis and control for predator-prey delays system. Chaos Solitons Fractals 123:361-372

19. Kirk KL (1998) Enrichment can stabilize population dynamics: autotoxins and density dependence. Ecol Soc Am 79(7):2456-2462

20. Holyoak M (2000) Effects of nutrient enrichment on predatorprey metapopulation dynamics. J Anim Ecol 69(6):985-997
21. Hadeler KP, Hillen $Y$ (2007) Coupled dynamics and quiescent states. Math Everywhere. Springer, Berlin, pp 7-23

22. De Stasio BT (1990) The role of dormancy and emergence patterns in the dynamics of a freshwater zooplankton community. Limnol Oceanogr 35(5):1079-1090

23. Davidowicz P, Gliwicz ZM, Gulati RD (1988) Can daphnia prevent a bluegreen algal bloom in hypertrophic lakes? A laboratory test. Limnologica 19(1):21-69

24. Dubey B, Kumar A, Maiti AP (2019) Global stability and Hopfbifurcation of prey-predator system with two discrete delays including habitat complexity and prey refuge. Commun Nonlinear Sci Numer Simul 67:528-554

25. Yang $Y(2009)$ Hopf bifurcation in a two-competitor, one-prey system with time delay. Appl Math Comput 214(1):228-235

26. Meng XY, Huo HF, Zhang XB, Xiang H (2011) Stability and Hopf bifurcation in a three-species system with feedback delays. Nonlinear Dyn 64(4):349-364

27. Juneja N, Agnihotri K, Kaur H (2018) Effect of delay on globally stable prey-predator system. Chaos Solitons Fract 111:146-156

28. Gopalsamy K (2013) Stability and oscillations in delay differential equations of population dynamics. Springer, Berlin

29. Cushing JM (1977) Integrodifferential equations and delay models in population dynamics. (Lecture notes in biomathematics). Springer, Berlin

30. Freedman HI, Rao VSH (1983) The trade-off between mutual interference and time lags in predator-prey systems. Bull Math Biol 45(6):991-1004

31. Rehim M, Imran M (2012) Dynamical analysis of a delay model of phytoplankton-zooplankton interaction. Appl Math Model 36(2):638-647

32. Das K, Ray S (2008) Effect of delay on nutrient cycling in phytoplankton-zooplankton interactions in estuarine system. Ecol Model 215(1-3):69-76

33. Gakkhar S, Singh A, Singh BP (2012) Effects of delay and seasonality on toxin producing phytoplankton-zooplankton system. Int J Biomath 5(5):1250047

34. Hastings A, Powell T (1991) Chaos in a three-species food chain. Ecology 72(3):896-903

35. Rai V, Upadhyay RK (2004) Chaotic population dynamics and biology of the top-predator. Chaos Solitons Fract 21(5):1195-1204

36. Gakkhar S, Naji RK (2005) Order and chaos in a food web consisting of a predator and two independent preys. Commun Nonlinear Sci Numer Simul 10(2):105-120

37. Liu W, Jiang Y (2018) Bifurcation of a delayed Gause predatorprey model with Michaelis-Menten type harvesting. JTheor Biol 438:116-132

38. Wang X, Peng M, Liu X (2015) Stability and Hopf bifurcation analysis of a ratio-dependent predator-prey model with two time delays and Holling type III functional response. Appl Math Comput 268:496-508

39. Rudman SM, Rodriguez-Cabal MA, Stier A, Sato T, Heavyside J, El-Sabaawi RW, Crutsinger GM (2015) Adaptive genetic variation mediates bottom-up and top-down control in an aquatic ecosystem. Proc R Soc B: Biol Sci 282(1812):20151234

40. Chatterjee A, Pal S, Chatterjee S (2011) Bottom up and top down effect on toxin producing phytoplankton and its consequence on the formation of plankton bloom. Appl Math Comput 218(7):3387-3398

41. Upadhyay RK, Thakur NK, Dubey B (2010) Nonlinear non-equilibrium pattern formation in a spatial aquatic system: effect of fish predation. J. Biol. Syst. 18(1):129-59

42. Chattopadhyay J, Sarkar RR, El Abdullaoui A (2002) A delay differential equation model on harmful algal blooms in the presence of toxic substances. Mathematical Medicine and Biology: A Journal of the IMA 19(2):137-161 
43. Zhao J, Wei J (2015) Dynamics in a diffusive plankton system with delay and toxic substances effect. Nonlinear Anal Real World Appl 22:66-83

44. Sharma A, Sharma AK, Agnihotri K (2014) The dynamic of plankton-nutrient interaction with delay. Appl Math Comput 231:503-515

45. Saha T, Bandyopadhyay M (2009) Dynamical analysis of toxin producing phytoplankton-zooplankton interactions. Nonlinear Anal: Real World Appl 10(1):314-332

46. Sharma A, Sharma AK, Agnihotri K (2016) Complex dynamic of plankton-fish interaction with quadratic harvesting and time delay. Model Earth Syst Environ 2(4):1-17

47. Pal S, Chatterjee A (2015) Dynamics of the interaction of plankton and planktivorous fish with delay. Cogent Math Stat 2(1):1074337

48. Liao T, Yu H, Zhao M (2017) Dynamics of a delayed phytoplankton-zooplankton system with Crowley-Martin functional response. Adv Differ Equ 1:5

49. Ruan S, Wei J (2001) On the zeros of a third degree exponential polynomial with applications to a delayed model for the control of testosterone secretion. Math Med Biol 18(1):41-52

50. Li MY, Shu H (2011) Multiple stable periodic oscillations in a mathematical model of CTL response to HTLV-I infection. Bull Math Biol 73(8):1774-1793

51. Hassard BD, Kazarinoff ND, Wan WH (1981) Theory and applications of Hopf bifurcation. Cambridge University Press, Cambridge
52. Kumar A, Srivastava PK, Yadav A (2019) Delayed information induces oscillations in a dynamical model for infectious disease. Int J Biomath 12(2):1950020

53. Raw SN, Mishra P (2018) Modeling and analysis of inhibitory effect in plankton-fish model: application to the hypertrophic Swarzedzkie Lake in Western Poland. Nonlinear Anal Real World Appl 46:465-492

54. Sharma A, Sharma AK, Agnihotri K (2015) Analysis of a toxin producing phytoplankton-zooplankton interaction with Holling IV type scheme and time delay. Nonlinear Dyn 81(1-2):13-25

55. Zhang Z, Rehim M (2017) Global qualitative analysis of a phytoplankton-zooplankton model in the presence of toxicity. Int J Dyn Control 5(3):799-810

56. Agrawal R, Jana D, Upadhyay RK, Rao VSH (2017) Complex dynamics of sexually reproductive generalist predator and gestation delay in a food chain model: double Hopf-bifurcation to Chaos. J Appl Math Comput 55(1-2):513-547

57. Pal N, Samanta S, Biswas S, Alquran M, Al-Khaled K, Chattopadhyay J (2015) Stability and bifurcation analysis of a three-species food chain model with delay. Int J Bifurc Chaos 25(9):1550123

Publisher's Note Springer Nature remains neutral with regard to jurisdictional claims in published maps and institutional affiliations. 